KIAS-P07001

\title{
One-loop Radiative Corrections to the $\rho$ Parameter in the Left Right Twin Higgs Model
}

\author{
Dong-Won Jung and Jae Yong Lee \\ School of physics, Korea Institute for Advanced Study, \\ 207-43, Cheongryangri-Dong, Dongdaemun-Gu, Seoul 130-722, Korea \\ E-mail: dwjung@kias.re.kr, littlehigg@kias.re.kr
}

\begin{abstract}
We implement a one-loop analysis of the $\rho$ parameter in the Left Right Twin Higgs model, including the logarithmically enhanced contributions from both fermion and scalar loops. Numerical results show that the one-loop contributions are dominant over the tree level corrections in most regions of parameter space. The experimentally allowed values of $\rho$-parameter divide the allowed parameter space into two regions; less than $670 \mathrm{GeV}$ and larger than $1100 \mathrm{GeV}$ roughly, for symmetry breaking scale $f$. Our numerical results significantly reduce the parameter space which are favorably accessible to the LHC.
\end{abstract}

Keywords: Left Right Twin Higgs model, $\rho$-parameter, LHC. 


\section{Introduction}

The Standard Model (SM) has excellently described high energy physics up to energies of $\mathcal{O}(100) \mathrm{GeV}$. The only undetected constituent of the SM, up to now, is a Higgs boson which is required to explain the generation of fermion and gauge boson masses. Theoretically the Higgs boson mass squared is quadratically sensitive to any new physics scale beyond the Standard Model (BSM) which may arise at higher energy scales and hence stabilization of the Higgs mass squared prefers the energy scale at which the BSM turns up to be lowered to $\mathcal{O}(1) \mathrm{TeV}$. On the other hand, electroweak precision measurements with naive naturalness assumption raise the energy scale of the BSM up to $100 \mathrm{TeV}$ or even higher. Hence, there remains a tension between theory and experiment associated with the stabilization of the SM Higgs mass. But with the start-up of the LHC in 2007 the tension may be relaxed by directly observing the BSM at TeV energy scale.

Supersymmetry is one of the most attractive approaches to stabilize the Higgs mass squared. The cancellation of quadratic divergences between the SM particle and their superpartner loops in the SM Higgs mass squared is one of the main motivation for the Minimal Supersymmetric Standard Model (MSSM) and its extensions. The MSSM predicts that the upper bound of the SM Higgs mass is $135 \mathrm{GeV}$ for $m_{t}=175 \mathrm{GeV}$ and $M_{S}=2 \mathrm{TeV}$, where

$M_{S}^{2} \equiv \frac{1}{2}\left(M_{\tilde{t}_{1}}^{2}+M_{\tilde{t}_{2}}^{2}\right)$ [1], 2]. This Higgs mass upper bound can be relaxed in non-minimal extensions of the MSSM. However, unsuppressed flavor changing neutral currents (FCNC) and other constraints almost rule out the entire parameter space of so-called MSSM-124 [3] in its most general form.

The idea of little Higgs originates in the speculation that the SM Higgs may be a pseudoNambu-Goldstone boson [4, 5, 6, 7, 8, 9, 10]. Stabilization of the Higgs mass in the little Higgs theories is achieved by the "collective symmetry breaking" which naturally renders the SM Higgs mass much smaller than the symmetry breaking scale. The distinct elements of little Higgs theories are a vector-like heavy top quark and various scalar and vector bosons. The former is universal while the latter is model-dependent. Both of them contributes significantly to one-loop processes and hence establish strict constraints on the parameter space of little Higgs theories. In the worst case, electroweak precision tests push up the symmetry breaking scale to $5 \mathrm{TeV}$ or higher, and regenerate significant fine-tuning in the Higgs potential.

The idea of twin Higgs shares the same origin with that of little Higgs in that the SM Higgs is a pseudo-Nambu-Goldstone boson [11]. But rather than using collective symmetry breaking to stabilize the Higgs mass squared, the twin Higgs mechanism makes use of additional discrete symmetry. In other words, the discrete symmetry ensures the absence of quadratic divergence in the Higgs mass squared. The mirror twin Higgs model containing a complete copy of the SM identifies the discrete symmetry with mirror parity. The SM world and its mirror world communicate only through the Higgs so that the mirror particles are very elusive in the SM world and yield poor phenomenology at the LHC.

The twin Higgs mechanism is also realized by identifying the discrete symmetry with leftright symmetry in the left-right model [12]. The left-right twin Higgs (LRTH) model contains 
$U(4)_{1} \times U(4)_{2}$ global symmetry as well as $S U(2)_{L} \times S U(2)_{R} \times U(1)_{B-L}$ gauge symmetry. The left-right symmetry acts on only the two $S U(2)$ 's gauge symmetry. A pair of vector-like heavy top quarks play a key role at triggering electroweak symmetry breaking just as that of the little Higgs theories. Besides, the other Higgs particles acquire large masses not only at quantum level but also at tree level. These heavy Higgs bosons make the model deliver much richer phenomenology at the LHC [13] compared with the mirror twin Higgs model. But theoretically they lead to large radiative corrections to one-loop processes and, in return, the allowed parameter space is reduced significantly. In this paper we perform a one-loop analysis of the $\rho$-parameter in the LRTH to reduce the parameter space.

The paper is organized as follows. The left right twin Higgs model is briefly reviewed in Section 2. The renormalization procedure for $\rho$-parameter is explained in Section 3. The numerical calculation for the $\rho$-parameter is performed in Section 4 . We present our conclusions in Section 5. The technical details for the computation of $\rho$-parameter is explained in Appendices.

\section{Left right twin Higgs model in a nutshell}

The LRTH model is based on the global $U(4)_{1} \times U(4)_{2}$ symmetry, with a locally gauged subgroup $S U(3)_{C} \times S U(2)_{L} \times S U(2)_{R} \times U(1)_{B-L}$. The two Higgs fields, $H$ and $\hat{H}$, are introduced and each transforms as $(4,1)$ and $(1,4)$ respectively under the global symmetry. They are written as

$$
H=\left(\begin{array}{c}
H_{L} \\
H_{R}
\end{array}\right), \quad \hat{H}=\left(\begin{array}{c}
\hat{H}_{L} \\
\hat{H}_{R}
\end{array}\right),
$$

where $H_{L, R}$ and $\hat{H}_{L, R}$ are two component objects which are charged under the $S U(2)_{L} \times$ $S U(2)_{R} \times U(1)_{B-L}$ as

$$
H_{L} \text { and } \hat{H}_{L}:(2,1,1), \quad H_{R} \text { and } \hat{H}_{R}:(1,2,1) .
$$

The global $U(4)_{1}\left(U(4)_{2}\right)$ symmetry is spontaneously broken down to its subgroup $U(3)_{1}\left(U(3)_{2}\right)$ with VEVs

$$
\langle H\rangle=\left(\begin{array}{c}
0 \\
0 \\
0 \\
f
\end{array}\right), \quad\langle\hat{H}\rangle=\left(\begin{array}{l}
0 \\
0 \\
0 \\
\hat{f}
\end{array}\right)
$$

Each spontaneous symmetry breaking results in seven Nambu-Goldstone bosons, which are parameterized as

$$
H=f e^{\pi / f}\left(\begin{array}{l}
0 \\
0 \\
0 \\
1
\end{array}\right), \quad \pi=\left(\begin{array}{cccc}
-\frac{N}{2 \sqrt{3}} & 0 & 0 & h_{1} \\
0 & -\frac{N}{2 \sqrt{3}} & 0 & h_{2} \\
0 & 0 & -\frac{N}{2 \sqrt{3}} & C \\
h_{1}^{*} & h_{2}^{*} & C^{*} & \frac{\sqrt{3} N}{2}
\end{array}\right),
$$


where $\pi$ is the corresponding Goldstone field matrix. $N$ is a neutral real field ${ }^{1}, C$ and $C^{*}$ are a pair of charged complex scalar fields, and $h_{S M}=\left(h_{1}, h_{2}\right)$ is the $\mathrm{SM} S U(2)_{L}$ Higgs doublet. $\hat{H}$ is parameterized in the identical way by its own Goldstone boson matrix, $\hat{\pi}$, which contains $\hat{N}, \hat{C}$, and $\hat{h}=\left(\hat{h}_{1}^{+}, \hat{h}_{2}^{0}\right)$. In turn, two $U(4) / U(3)$ 's symmetry breaking left with fourteen Nambu-Goldstone bosons.

The linear combination of $C$ and $\hat{C}$, and the linear combination of $N$ and $\hat{N}$ are eaten by the gauge bosons of $S U(2)_{R} \times U(1)_{B-L}$, which is brken down to the $U(1)_{Y}$. The orthogonal linear combinations, a charged complex scalar $\phi^{ \pm}$and a neutral real pseudoscalar $\phi^{0}$, remain as Nambu-Goldstone bosons. On top of that, the SM Higgs acquires a VEV $\left\langle h_{S M}\right\rangle=$ $(0, v / \sqrt{2})$, and thereby electroweak symmetry $S U(2)_{L} \times U(1)_{Y}$ is broken down to $U(1)_{E M}$. But $h$ 's do not get a VEV and remain as Nambu Goldstone bosons. At the end of the day, the two Higgs VEVs are given by

$$
\langle H\rangle=\left(\begin{array}{c}
0 \\
\text { if } \sin x \\
0 \\
f \cos x
\end{array}\right), \quad\langle\hat{H}\rangle=\left(\begin{array}{l}
0 \\
0 \\
0 \\
\hat{f}
\end{array}\right)
$$

where $x=v /(\sqrt{2} f)$. The values of $f$ and $\hat{f}$ will be bounded by electroweak precision measurements. In addition, $f$ and $\hat{f}$ are interconnected once we set $v=246 \mathrm{GeV}$.

\subsection{Gauge sector}

The whole gauge symmetry of the model is $S U(3)_{C} \times S U(2)_{L} \times S U(2)_{R} \times U(1)_{B-L}$. But since $S U(3)_{C}$ is irrelevant to eletroweak symmetry breaking $S U(3)_{C}$ gauge symmetry is not taken into account in this paper. The generators of the $S U(2)_{L} \times S U(2)_{R} \times U(1)_{B-L}$ is given respectively as,

$$
\left(\begin{array}{cc}
\frac{1}{2} \sigma_{i} & 0 \\
0 & 0
\end{array}\right), \quad\left(\begin{array}{cc}
0 & 0 \\
0 & \frac{1}{2} \sigma_{i}
\end{array}\right), \quad \frac{1}{2}\left(\begin{array}{cc}
1_{2} & 0 \\
0 & 1_{2}
\end{array}\right),
$$

and the corresponding gauge fields are $W_{L}^{ \pm, 0}, W^{ \pm, 0}$ and $B$, respectively. The covariant derivative is then given as ${ }^{2}$

$$
D_{\mu}=\partial_{\mu}-i g \mathcal{W}_{\mu}-i g^{\prime} q_{B-L} \mathcal{B}_{\mu}
$$

where

$$
\mathcal{W}=\frac{1}{2}\left(\begin{array}{cccc}
W_{L}^{0} & \sqrt{2} W_{L}^{+} & 0 & 0 \\
\sqrt{2} W_{L}^{-} & -W_{L}^{0} & 0 & 0 \\
0 & 0 & W_{R}^{0} & \sqrt{2} W_{R}^{+} \\
0 & 0 & \sqrt{2} W_{R}^{-} & -W_{R}^{0}
\end{array}\right), \quad \mathcal{B}=\frac{1}{2}\left(\begin{array}{cccc}
B & 0 & 0 & 0 \\
0 & B & 0 & 0 \\
0 & 0 & B & 0 \\
0 & 0 & 0 & B
\end{array}\right) \text {, }
$$

and $g$ and $g^{\prime}$ are the gauge couplings for $S U(2)_{L, R}$ and $U(1)_{B-L}$, and $q_{B-L}$ is the charge of the field under $U(1)_{B-L}$.

\footnotetext{
${ }^{1}$ The normalization is naturally altered when applying the unitary gauge.

${ }^{2}$ Note that due to the left-right parity the gauge coupling of $S U(2)_{L}$ is equal to that of $S U(2)_{R}$.
} 
The kinetic term for the two Higgs fields can be written as

$$
\mathcal{L}_{H}=\left(D_{\mu} H\right)^{\dagger} D^{\mu} H+\left(D_{\mu} \hat{H}\right)^{\dagger} D^{\mu} \hat{H}
$$

with $q_{B-L}=1$. As previously said, the Higgs mechanism for both $H$ and $\hat{H}$ makes the six gauge bosons massive whereas one gauge boson, photon, massless. For the charged gauge bosons, there is no mixing between the $W_{L}^{ \pm}$and $W_{R}^{ \pm}: W_{L}^{ \pm}$is identified with the SM weak gauge boson $W^{ \pm}$while $W_{R}^{ \pm}$is much heavier than $W^{ \pm}$and is denoted as $W_{H}^{ \pm}$. Their masses are

$$
\begin{aligned}
M_{W}^{2} & =\frac{1}{2} g^{2} f^{2} \sin ^{2} x, \\
M_{W_{H}}^{2} & =\frac{1}{2} g^{2}\left(\hat{f}^{2}+f^{2} \cos ^{2} x\right) .
\end{aligned}
$$

Note that $M_{W}^{2}+M_{W_{H}}^{2}=\frac{g^{2}}{2}\left(f^{2}+\hat{f}^{2}\right)$. The linear combinations of the neutral gauge bosons $W_{L}^{0}, W_{R}^{0}$ and $B$ yield two neutral massive gauge bosons $Z, Z_{H}$ and one photon $A$ with masses, respectively:

$$
\begin{aligned}
M_{A}^{2} & =0 \\
M_{Z}^{2} & =\frac{g^{2}+2 g^{\prime 2}}{g^{2}+g^{\prime 2}} \frac{2 M_{W}^{2} M_{W_{H}}^{2}}{M_{W_{H}}^{2}+M_{W}^{2}+\sqrt{\left(M_{W_{H}}^{2}-M_{W}^{2}\right)^{2}+4 \frac{g^{\prime 2}}{g^{2}+g^{\prime 2}} M_{W_{H}}^{2} M_{W}^{2}}}, \\
M_{Z_{H}}^{2} & =\frac{g^{2}+g^{\prime 2}}{g^{2}}\left(M_{W}^{2}+M_{W_{H}}^{2}\right)-M_{Z}^{2} .
\end{aligned}
$$

For later use, we define the Weinberg angle in the LRTH model:

$$
\begin{gathered}
s_{w}=\sin \theta_{w}=\frac{g^{\prime}}{\sqrt{g^{2}+2 g^{\prime 2}}}, \\
c_{w}=\cos \theta_{w}=\sqrt{\frac{g^{2}+g^{\prime 2}}{g^{2}+2 g^{\prime 2}}} \\
c 2_{w}=\sqrt{\cos 2 \theta}=\frac{g}{\sqrt{g^{2}+2 g^{\prime 2}}} .
\end{gathered}
$$

The unit of the electric charge is then given by

$$
e=g s_{w}=\frac{g g^{\prime}}{\sqrt{g^{2}+2 g^{\prime 2}}}
$$

\subsection{Fermion sector}

To cancel the quadratic sensitivity of the Higgs mass to the top quark loops, a pair of vectorlike, charge $2 / 3$ fermion $\left(\mathcal{Q}_{L}, \mathcal{Q}_{R}\right)$ are incorporated into the top Yukawa sector,

$$
\mathcal{L}_{Y u k}=y_{L} \bar{Q}_{L 3} \tau_{2} H_{L}^{*} \mathcal{Q}_{R}+y_{R} \bar{Q}_{R 3} \tau_{2} H_{R}^{*} \mathcal{Q}_{L}-M \overline{\mathcal{Q}}_{L} \mathcal{Q}_{R}+\text { h.c. }
$$


where $\tau_{2}=\left(\begin{array}{cc}0 & -1 \\ 1 & 0\end{array}\right), Q_{L 3}=-i\left(u_{L 3}, d_{L 3}\right)$ and $Q_{R 3}=\left(u_{R 3}, d_{R 3}\right)$ are the third generation upand down-type quarks, respectively. The left-right parity indicates $y_{L}=y_{R}(\equiv y)$. The mass parameter $M$ is essential to the top mixing. The value of $M$ is constrained by the $Z \rightarrow b \bar{b}$ branching ratio. It can be also constrained by the oblique parameters, which we will do in the letter. Furthermore, it yields large log divergence of the SM Higgs mass. To compensate for it the heavy gauge bosons also get large masses by increasing the value of $\hat{f}$. Therefore it is natural for us to take $M \lesssim y f$.

Expanding the $H_{L, R}$ field in terms of Nambu-Goldstone boson fields, we acquire the mass matrix of the fermions. By diagonalizing it we obtain not only the mass eigenstates for the SM-like and heavy top quarks, but also the mixing angles for the left-handed and right-handed fermions, as follows,

$$
\begin{aligned}
m_{t}^{2} & =\frac{1}{2}\left(M^{2}+y^{2} f^{2}-N_{t}\right), \\
m_{T}^{2} & =\frac{1}{2}\left(M^{2}+y^{2} f^{2}+N_{t}\right), \\
\sin \alpha_{L} & =\frac{1}{\sqrt{2}} \sqrt{1-\left(y^{2} f^{2} \cos 2 x+M^{2}\right) / N_{t}}, \\
\sin \alpha_{R} & =\frac{1}{\sqrt{2}} \sqrt{1-\left(y^{2} f^{2} \cos 2 x-M^{2}\right) / N_{t}},
\end{aligned}
$$

where $N_{t}=\sqrt{\left(y^{2} f^{2}+M^{2}\right)^{2}-y^{4} f^{4} \sin ^{2} 2 x}$.

\subsection{Revisit of Higgs sector}

Among the fourteen Nambu-Goldstone bosons in both $\pi$ and $\hat{\pi}$, six Nambu-Goldstone bosons are eaten by the gauge bosons. The remaining eight Nambu-Goldstone bosons acquire masses through quantum effects and/or soft symmetry breaking terms, so called " $\mu$-term". The Coleman-Weinberg potential, obtained by integrating out the gauge bosons and top quarks, yields the SM Higgs potential, which determines the SM Higgs VEV and its mass, as well as the masses for the other Higgs, $\phi^{ \pm}, \phi^{0}, \hat{h}_{1}^{ \pm}$and $\hat{h}_{2}^{0}$. On the other hand, the $\mu$-term ${ }^{3}$,

$$
V_{\mu}=-\mu_{r}^{2}\left(H_{R}^{\dagger} \hat{H}_{R}+\text { c.c. }\right)+\hat{\mu}^{2} \hat{H}_{L}^{\dagger} \hat{H}_{L}
$$

\footnotetext{
${ }^{3}$ In the potential, $-\mu_{l}^{2}\left(H_{L}^{\dagger} \hat{H}_{L}+\right.$ c.c. $)$ is possible, but we choose $\mu_{l}=0$ for not spoiling the original motivation of the model and preserving the stability of $\hat{h}_{2}$ dark matter [13.
} 
contributes to the Higgs masses at tree level. Here we write down the masses for the Higgs.

$$
\begin{aligned}
M_{\phi^{0}}^{2}= & \frac{\mu_{r}^{2} f \hat{f}}{\hat{f}^{2}+f^{2} \cos ^{2} x}\left[\frac{\hat{f}^{2}\left(\cos x+\frac{\sin x}{x}\left(4+x^{2}\right)\right)}{f^{2}\left(\cos x+2 \frac{\sin x}{x}\right)^{2}}+\frac{2 \cos x\left(\cos x+4 \frac{\sin x}{x}\right)}{3\left(\cos x+2 \frac{\sin x}{x}\right)}\right. \\
& \left.+\frac{f^{2} \cos ^{2} x(4+\cos x)}{9 \hat{f}^{2}}\right], \\
M_{\phi^{ \pm}}^{2}= & \frac{3}{16 \pi^{2}} \frac{g^{2} M_{W_{H}}^{2}}{M_{Z_{H}}^{2}-M_{Z}^{2}}\left[\left(\frac{M_{W}^{2}}{M_{Z_{H}}^{2}}-1\right) \mathcal{Z}\left(M_{Z_{H}}\right)-\left(\frac{M_{W}^{2}}{M_{Z}^{2}}-1\right) \mathcal{Z}\left(M_{Z}\right)\right] \\
& +\frac{\mu_{r}^{2} f \hat{f}}{\hat{f}^{2}+f^{2} \cos ^{2} x}\left[\frac{\hat{f}^{2} x^{2}}{f^{2} \sin ^{2} x}+2 \cos x+\frac{f^{2} \cos ^{3} x}{\hat{f}^{2}}\right], \\
M_{\hat{h}_{2}}^{2}= & \frac{3}{16 \pi^{2}}\left[\frac{g^{2}}{2}\left(\mathcal{Z}\left(M_{W}\right)-\mathcal{Z}\left(M_{W_{H}}\right)\right)+\frac{2 g^{2}+g^{2}}{4} \frac{M_{W_{H}}^{2}-M_{W}^{2}}{M_{Z_{H}}^{2}-M_{Z}^{2}}\left(\mathcal{Z}\left(M_{Z}\right)-\mathcal{Z}\left(M_{Z_{H}}\right)\right)\right] \\
& +\mu_{r}^{2} \frac{f}{\hat{f}} \cos x+\hat{\mu}^{2}, \\
M_{\hat{h}_{1}}^{2}= & M_{\hat{h}_{2}}^{2}+\frac{3}{16 \pi^{2}} \frac{g^{\prime 2} M_{W}^{2}}{M_{Z_{H}}^{2}-M_{Z}^{2}}\left[\left(\frac{M_{W_{H}}^{2}}{M_{Z_{H}}^{2}}-1\right) \mathcal{Z}\left(M_{Z_{H}}\right)-\left(\frac{M_{W_{H}}^{2}}{M_{Z}^{2}}-1\right) \mathcal{Z}\left(M_{Z}\right)\right] .
\end{aligned}
$$

where

$$
\mathcal{Z}(x)=-x^{2}\left(\ln \frac{\Lambda^{2}}{x^{2}}+1\right) .
$$

The SM Higgs potential arises mainly from both top sector and gauge sector. The contribution of fermion loop to the SM Higgs mass squared is negative and its dominance over the contribution of gauge boson loops and tree level mass parameter $\mu_{r}^{2} \frac{\hat{f}}{2 f}$ triggers electroweak symmetry breaking. We fix the SM Higgs VEV, $v=246 \mathrm{GeV}$.

\section{The renormalization procedure ${ }^{4}$}

The $Z$-pole, $W$-mass, and neutral current data can be used to search for and set limits on deviations from the SM. Many extensions of the SM are described by the $\rho$-parameter, which is defined as

$$
\rho \equiv \frac{M_{W}^{2}}{M_{Z}^{2} c_{\theta}^{2}}
$$

The effective leptonic mixing angle $s_{\theta}^{2}\left(=1-c_{\theta}^{2}\right)$ at the $Z$-resonance is defined as the ratio of the electron vector to axial vector coupling constants to the $Z$-boson,

$$
\frac{\operatorname{Re}\left(g_{V}^{e}\right)}{\operatorname{Re}\left(g_{A}^{e}\right)} \equiv 4 s_{\theta}^{2}-1
$$

where the coupling constants of a fermion $\psi$ to the gauge boson $X$ is given as,

$$
\mathcal{L}=i \bar{\psi}_{1} \gamma_{\mu}\left(g_{V}+g_{A} \gamma_{5}\right) \psi_{2} X^{\mu}
$$

\footnotetext{
${ }^{4}$ In this section, we keep the track of the discussion in Ref. 14]
} 
The effective Lagrangian of the charged current interaction in the LRTH model is given by

$$
\mathcal{L}_{c c}=\frac{g}{\sqrt{2}}\left(W_{\mu L}^{+} J_{L}^{\mu-}+W_{\mu L}^{-} J_{L}^{\mu+}\right)+(L \rightarrow R),
$$

where $J_{L, R}^{\mu \pm}$ is the charged currents. For momenta quite small compared to $M_{W}$, this effective Lagrangian gives rise to the effective four-fermion interaction with the Fermi coupling constant,

$$
\frac{G_{F}}{\sqrt{2}}=\frac{g^{2}}{8 M_{W}^{2}}
$$

and the vector and axial vector parts of the neutral current Zee coupling constants are given to the order $v^{2} / \hat{f}^{2}$ as,

$$
\begin{aligned}
g_{V}^{e} & =\frac{g}{2 c_{w}}\left[\left(-\frac{1}{2}+2 s_{w}^{2}\right)+\frac{v^{2}}{4\left(f^{2}+\hat{f}^{2}\right)} \frac{s_{w}^{2}\left(c 2_{w}^{2}-2\right)}{c_{w}^{4}}\right], \\
g_{A}^{e} & =\frac{g}{2 c_{w}}\left[\frac{1}{2}+\frac{v^{2}}{4\left(f^{2}+\hat{f}^{2}\right)} \frac{s_{w}^{2} c 2_{w}^{2}}{c_{w}^{4}}\right] .
\end{aligned}
$$

The effective leptonic mixing angle $s_{\theta}^{2}$ is then related to the mixing angle $s_{w}^{2}$ as

$$
s_{\theta}^{2}=s_{w}^{2}+\frac{v^{2}}{4\left(f^{2}+\hat{f}^{2}\right)} \frac{s_{w}^{2}\left(c 2_{w}^{2}-2 s_{w}^{2}\right)}{c_{w}^{2}} .
$$

It can then be inverted and gives

$$
s_{w}^{2}=s_{\theta}^{2}+\Delta s_{\theta}^{2}
$$

where

$$
\frac{\Delta s_{\theta}^{2}}{s_{\theta}^{2}}=-\frac{1}{4 \sqrt{2} G_{F}\left(f^{2}+\hat{f}^{2}\right)} \frac{1-3 s_{\theta}^{2}}{c_{\theta}^{2}} .
$$

The SM $S U(2)_{L}$ gauge coupling constant, $g$, can be expressed by the effective leptonic mixing angle, $s_{\theta}^{2}$, and the fine-structure constant, $\alpha$, as

$$
g^{2}=\frac{e^{2}}{s_{w}^{2}}=\frac{4 \pi \alpha}{s_{\theta}^{2}}\left(1-\frac{\Delta s_{\theta}^{2}}{s_{\theta}^{2}}\right)
$$

The $\rho$-parameter at the tree level is

$$
\rho^{\text {tree }}=\frac{\pi \alpha}{\sqrt{2} G_{F} c_{\theta}^{2} s_{\theta}^{2} M_{Z}^{2}}\left(1-\frac{\Delta s_{\theta}^{2}}{s_{\theta}^{2}}\right) .
$$

Note that the $\rho$-parameter is different from unity already at the tree level and its deviation from unity is of order $v^{2} / \hat{f}^{2}$.

Since the loop factor occurring in radiative corrections, $1 / 16 \pi^{2}$, is similar in magnitude to $v^{2} / \hat{f}^{2}$ (for $\hat{f} \gtrsim 5 \mathrm{TeV}$ ), the one-loop radiative corrections can be comparable in size to the next-to-leading order contributions at tree level. At one-loop the mass relation reads [15]

$$
s_{\theta}^{2} c_{\theta}^{2}=\frac{\pi \alpha\left(M_{Z}\right)}{\sqrt{2} G_{F} M_{Z}^{2} \rho}\left[1-\frac{\Delta s_{\theta}^{2}}{s_{\theta}^{2}}+\Delta r_{Z}\right],
$$


where $\Delta r_{Z}$ includes radiative effects from various sources:

$$
\Delta r_{Z}=\frac{\delta \alpha}{\alpha}-\frac{\delta G_{F}}{G_{F}}-\frac{\delta M_{Z}^{2}}{M_{Z}^{2}}-\left(\frac{c_{\theta}^{2}-s_{\theta}^{2}}{c_{\theta}^{2}}\right) \frac{\delta s_{\theta}^{2}}{s_{\theta}^{2}} .
$$

Note that $\Delta r_{Z}$ defined in Eq. (3.14) differs from the usual $\Delta \hat{r}_{Z}$ defined in the SM by an extra contributions due to the renormalization of $s_{\theta}^{2}$. In general, The vertex and box contributions to the radiative effects are relatively small compared to the other corrections [15, 16, 17] and hence we consider only the so-called "oblique" type, i.e. the $W$-, $Z$ - and $\gamma$-propagators. The contribution due to the vacuum polarization of the photon, $\delta \alpha$, is given by

$$
\frac{\delta \alpha}{\alpha}=\Pi^{\gamma \gamma^{\prime}}(0)+2\left(\frac{g_{V}^{e}-g_{A}^{e}}{Q_{e}}\right) \frac{\Pi^{\gamma Z}(0)}{M_{Z}^{2}} .
$$

Since we ignore the vertex and box corrections, the electroweak radiative correction to the Fermi constant, $\delta G_{F}$, stems from the $W$-boson vacuum polarization,

$$
\frac{\delta G_{F}}{G_{F}}=-\frac{\Pi^{W W}(0)}{M_{W}^{2}}
$$

The counterterms for the $Z$-boson mass, $\delta M_{Z}^{2}$, and the leptonic mixing angle, $\delta s_{\theta}^{2}$, are given by, respectively 16]

$$
\begin{aligned}
& \delta M_{Z}^{2}=\operatorname{Re}\left(\Pi^{Z Z}\left(M_{Z}^{2}\right)\right) \\
& \frac{\delta s_{\theta}^{2}}{s_{\theta}^{2}}=R e\left[( \frac { c _ { \theta } } { s _ { \theta } } ) \left[\frac{\Pi^{\gamma Z}\left(M_{Z}^{2}\right)}{M_{Z}^{2}}+\frac{v_{e}^{2}-a_{e}^{2}}{a_{e}} \Sigma_{A}^{e}\left(m_{e}^{2}\right)\right.\right. \\
& \left.\left.-\frac{v_{e}}{2 s_{\theta} c_{\theta}}\left(\frac{\Lambda_{V}^{Z \bar{e} e}\left(M_{Z}^{2}\right)}{v_{e}}-\frac{\Lambda_{A}^{Z \bar{e} e}\left(M_{Z}^{2}\right)}{a_{e}}\right)\right]\right],
\end{aligned}
$$

where $\Lambda_{V, A}^{Z \bar{e} e}$ are the vector and axial vector form factors of the unnormalized one-loop Zee vertex corrections, and $\Sigma_{A}^{e}$ is the axial part of the electron self-energy. Once again, we ignore these "non-oblique" type contributions.

Defining a short-hand notation $\Delta \hat{r}$,

$$
\Delta \hat{r}=-\frac{\Delta s_{\theta}^{2}}{s_{\theta}^{2}}-\frac{\operatorname{Re}\left(\Pi^{Z Z}\left(M_{Z}^{2}\right)\right)}{M_{Z}^{2}}+\Pi^{\gamma \gamma^{\prime}}(0)+2\left(\frac{g_{V}^{e}-g_{A}^{e}}{Q_{e}}\right) \frac{\Pi^{\gamma Z}(0)}{M_{Z}^{2}}-\frac{c_{\theta}^{2}-s_{\theta}^{2}}{c_{\theta} s_{\theta}} \frac{\operatorname{Re}\left(\Pi^{\gamma Z}\left(M_{Z}^{2}\right)\right)}{M_{Z}^{2}},
$$

we can then rewrite Eq. (3.13) as,

$$
s_{\theta}^{2} c_{\theta}^{2}=\frac{\pi \alpha\left(M_{Z}\right)}{\sqrt{2} G_{F} M_{Z}^{2} \rho}\left[1+\frac{\Pi^{W W}(0)}{M_{W}^{2}}+\Delta \hat{r}\right] .
$$

Solving for $M_{W}^{2}$ and $\rho$, we make a prediction for the physical $W$-boson mass

$$
M_{W}^{2}=\frac{1}{2}\left[a(1+\Delta \hat{r})+\sqrt{a^{2}(1+\Delta \hat{r})^{2}+4 a \Pi^{W W}(0)}\right],
$$

with $\quad a \equiv \frac{\pi \alpha\left(M_{Z}\right)}{\sqrt{2} G_{F} s_{\theta}^{2}}$. The $\rho$ parameter is then obtained using Eq. (3.1) with the $M_{W}^{2}$ value predicted in Eq. (3.21). 


\section{Numerical Analysis}

For doing the calculation concerning the precision measurements, the standard experimental values are necessary which play as input parameters. Here, we use the following experimentally measured values for the input parameters [18, 20]:

$$
\begin{aligned}
G_{F} & =1.16637(1) \times 10^{-5} \mathrm{GeV}^{-2}, \\
M_{Z} & =91.1876(21) \mathrm{GeV} \\
\alpha\left(M_{Z}\right)^{-1} & =127.918(18) \\
s_{\theta}^{2} & =0.23153(16) .
\end{aligned}
$$

We also take the top and bottom quark masses as 18, 19,

$$
m_{t}=172.3 \mathrm{GeV}, \quad m_{b}=3 \mathrm{GeV},
$$

where $m_{t}$ is the central value of the electroweak fit and $m_{b}$ is the running mass at the $M_{Z}$ scale with $\bar{M} S$ scheme. The $\rho$-parameter itself is measured very accurately. Including the all SM corrections (top quark contribution, bosonic loop correction et.al.,), the observed range is 18

$$
1.00989 \leq \rho^{\exp } \leq 1.01026 .
$$

The input parameters of the LRTH model [12] are as follows;

$$
f, M, \mu_{r}, \hat{\mu},
$$

where $M$ is the heavy top quark mass scale, both $\mu_{r}$ and $\hat{\mu}$ are soft symmetry breaking terms. The masses of the top and heavy top quarks are determined by $f$ and $M$ while those of the scalar particles $\hat{h}_{1}, \hat{h}_{2}, \phi^{ \pm}$and $\phi^{0}$ largely depend on $\hat{\mu}, \mu_{r}$ and $f$. Another scale $\hat{f}$, which is associated with the masses of the heavy gauge bosons, can be determined from the electroweak symmetry breaking condition: there is a generic relation between $\hat{f}$ and $f$ since Coleman-Weinberg potential of the Higgs boson mostly depends on $M, f$ and $\hat{f}$. For scalar potential, there is a tree level mass term proportional to $\mu_{r}^{2}$. So we may not acquire negative mass squared term which is necessary for electroweak symmetry breaking and it gives an upper bound for the value of $\mu_{r}$.

We plot in Fig. $1 \hat{f}$ versus $f$ with various values of $M$. For a given $f, \hat{f}$ becomes larger as $M$ increases. It is because the increase of $M$ contributes positively to the Higgs mass through the top loop while the increase of $\hat{f}$ contributes negatively to the Higgs mass through the gauge boson loop, and thereby these contributions cancel out themselves in order to retain $v=246 \mathrm{GeV}$. Of course there is a contribution from tree level mass term $\mu_{r}^{2}$, but in most cases it cause little difference to the relations appear in the Fig.11. This insensitiveness can be figured out with simple evaluation. First, from the electroweak symmetry breaking condition, the mass squared contribution from the soft symmetry breaking term $\mu_{r}^{2} \frac{\hat{f}}{2 f}$ should be smaller 
than that from the fermion loop. This can be written down approximately as follows:

$$
\mu_{r}^{2} \frac{\hat{f}}{2 f}<\frac{3}{8 \pi^{2}}\left(M^{2}+y^{2} f^{2}\right) .
$$

In the above inequality, we ignore the gauge boson loop contributions since they are small compared to the fermion loop contributions. In general $\hat{f}$ is larger than $f$ about 5 times or more and $\frac{3}{8 \pi^{2}}$ is very small, we can see that $\mu_{r}$ should be very much smaller than $f$. To get the $\hat{f}$ which reproduces the electroweak symmetry breaking scale $v=246 \mathrm{GeV}$, we should solve the equation,

$$
\frac{3 g^{4}}{64 \pi^{2}} f \hat{f}^{2}+\mu_{r}^{2} \hat{f}+2 \lambda v^{2} f-\frac{3}{4 \pi^{2}} f\left(M^{2}+f^{2}\right)+\frac{3 g^{4}}{64 \pi^{2}}=0
$$

for given $f, M$ and $\mu_{r} . \lambda$ in the above equation is the coefficient of quartic term and less than 1 in general. Note that we derive the above equation with some degree of approximation. For example, we ignore the logarithmic terms. But the crude behavior will be similar. In this equation, the coefficients of $\hat{f}^{2}$ and $\hat{f}$ are much smaller than constant term, so the solution $\hat{f}$ is almost insensitive to the value of $\mu_{r} .{ }^{5}$

Plots in Fig. 2 illustrates the behavior of one-loop $\rho$-parameter calculated with various values of $M$. For the case of $M=0$, where there is no mixing between the top and heavy top quarks, $\Delta \rho$ increases monotonically with $f$. But for nonzero $M$ where the mixing is turned on, mass of the heavy top quark become large as $M$ increases for a given $f$, and the fermionic loop contributions tend to become large, too. But at the same time the effects of mixing angles on the fermionic loops become significant as either $f$ or $M$ increases while the condition of electroweak symmetry breaking is retained. In other words, since the mixing angles are determined by $f$ and $M$, the one-loop corrected $\rho$ parameter begins to wobble as $f$ increases even with the fixed scalar mass parameters. Because of this nature, fine tuning in the $\rho$-parameter is inevitable for large $f$, as will be shown later.

To draw a meaningful information on the model parameters from the $\rho$-parameter, we scan the parameter space generally, i.e.,

$$
500 \mathrm{GeV} \leq f \leq 2500 \mathrm{GeV}, \quad 0 \leq M, \mu_{r}, \hat{\mu} \leq f
$$

Even though too large $f$ makes the model unviable, we take the rather large value of $f$, $2.5 \mathrm{TeV}$, as an upper limit for completeness of the scanning. As a result of $\rho$-parameter calculation, we can obtain the allowed regions of parameter space. As an example, Fig. 3 shows the allowed regions of parameter space (a) for $f$ versus $M$ and (b) for $f$ versus $\mu_{r}$. It is interesting to notice that the allowed parameter space is divided into two regions; less than $670 \mathrm{GeV}$ and larger than $1100 \mathrm{GeV}$ roughly, for $f$. This can be figured out as follows. The loop corrections tend to be larger as $f$ increases. It is because the masses of the particles

\footnotetext{
${ }^{5}$ If we rewrite the equation as $a \hat{f}^{2}+b \hat{f}+c=0$, the inequality $a, b \ll c$ is satisfied. In this case the solution is $\hat{f} \simeq \sqrt{\frac{c}{a}}-\frac{b}{a} \simeq \sqrt{\frac{c}{a}}$.
} 
involved in one-loop correction increase in general as $f$ increases. But at the same time, the mixing angles of top-heavy top quarks also vary. Since the mixing angles depend on not only $f$ but also $M$, these two effects compete during the increase of $f$. Because of this interplay of top mixing angles and masses, we have two distinct allowed parameter spaces. For small $f$, solution points prefer very small values of $M$. It means there is no large mixing between the top and heavy top quarks. In general, $\Pi^{W W}(0)$ is large for small $f$, and decreases as $f$ increases. So for fitting the observed W-boson mass in the small $f$ region, which is directly related to the $\rho$-parameter, we restrict the $\Delta \hat{r}$ within rather small range. Because the $\Delta \hat{r}$ is mostly determined by $\Pi^{Z Z}\left(M_{Z}^{2}\right)$, it should be also small. For doing that, we should take the small value of $M$, which makes the masses and mixing angles of heavy top quark small. We find that in the small $f$ region, $M$ should be smaller than about $22 \mathrm{GeV}$. Soft symmetry breaking parameter $\mu_{r}$ is restricted to the values less than around $60 \mathrm{GeV}$. This bound arises mainly from the electroweak symmetry breaking condition, and is generically independent of the $\rho$-parameter. Another free parameters $\hat{\mu}$ is not restricted from the one-loop corrected $\rho$-parameter. The reason is that $\hat{\mu}$ only contributes to the masses of $\hat{h}_{1}$ and $\hat{h}_{2}$, and their contributions are effectively cancelled among the relevant loop diagrams. It is pointed out in the appendix $\mathrm{C}$.

This region of parameter space can provide constraints on the masses of many particles appear in this model. First, let us consider the masses of the heavy top and heavy gauge bosons. As shown in Fig. 4, their masses generically increase as $f$ increases. The mass of the heavy top quark is uniquely determined when $f, \hat{f}$ and $M$ are fixed. So does top Yukawa coupling. Basically $\hat{f}$ is determined by the electroweak symmetry breaking condition, but their $M$ and $\mu_{r}$ dependence provokes the ambiguity on its value. For small $f$ region, since $M$ is also very small, the $M_{T}$ is almost determined by $f$ alone. It appears as straight line in Fig. 1 (a). For large $f$ region, it becomes spread due to the top mixing angles. The plots of the heavy $Z$ and $W$ boson masses versus $f$ are quite similar to that of the heavy top mass versus $f$. In the case of heavy $W$ boson, the strongest constraint comes from $K_{L}-K_{S}$ mixing. The strongest bound to date is $m_{W_{H}}>1.6 \mathrm{TeV}$, with the assumption of $g_{L}=g_{R}$ and $V_{L}=V_{R}\left(V_{R}^{*}\right)$. [25, 26] This can exclude some region from Fig. [4(c). In this case, we can place the lower bound on $f$ such that $660 \mathrm{GeV}$. If the lower bound for $m_{W_{H}}$ is pushed up over $1750 \mathrm{GeV}$, the small $f$ region is completely excluded and so we can give the lower bound for $f$ as $1.1 \mathrm{TeV}$ from our calculation of the $\rho$-parameter and for many particles appear in the model as well. But in this paper we do not include the mechanism of neutrino oscillation and the quark mixing structure, so the restriction on $m_{W_{H}}$ can be relaxed. Other constraints on the $m_{W_{H}}$ from the direct searches at CDF and D0 are about $650 \sim 786 \mathrm{GeV}$, as lower bound. 27, 28] Our results remain safe from these experimental bounds. The heavy $Z$ boson has also been studied in detail by many experimentalists. The current experimental bound is about $500 \sim 800 \mathrm{GeV}$ from the precision measurements [18] and $\sim 630 \mathrm{GeV}$ from CDF [18]. In this case, also safe is the mass of heavy $Z$ boson.

With the parameters allowed by the $\rho$-parameter, the masses of new scalar bosons $\hat{h}_{1,2}, \phi^{0}$ and $\phi^{ \pm}$are also constrained. $\hat{h}_{1,2}$ has almost degenerate masses, and are dependent on both 
$\mu_{r}$ and $\hat{\mu}$, unlike the $\phi^{0, \pm}$ which depend only on $\mu_{r}$. Their masses are severely constrained according to the value of $f$. Unfortunately, we cannot give a lower bound on the mass of $\phi^{0}$. In fact, its mass, though it is quite small, arise from radiative corrections. For $\phi^{ \pm}$, the loop contribution is rather large so it acquires larger mass compared to the neutral one. Fig. 5 shows those allowed regions. The $\rho$-parameter cannot give a strong restriction on the Higgs mass. In the whole space, Higgs mass is restricted below about $167 \mathrm{GeV}$. We cannot give a lower bound for Higgs boson mass from $\rho$-parameter itself. Here, we adopt the LEP bound for Higgs mass, $114.4 \mathrm{GeV}$ 29], since its structure is same as the SM. The generic behavior of Higgs mass as a function of $f$ is shown in Fig .6.

We can summarize the results of our analysis as follows. With the observed $\rho$-parameter, we can divide the allowed region into two pieces. $f$ smaller than about $670 \mathrm{GeV}$ and larger than about $1.1 \mathrm{TeV}$. We give the bounds on the mass spectrum of many particles for each region. Especially the heavy gauge bosons remain safe from the experimental constraints. Unlike the other particles, we cannot give a lower bound for $\phi^{0}$. But loop correction play an important role for $\phi^{ \pm}$, generate the mass difference between charged and neutral scalars. Another analyses are necessary to determined which region is allowed. If the small $f$ region is excluded, for example by the authors of Ref. [13], we can provide exact lower bounds for

the masses of $T, Z_{H}, W_{H}, \hat{h}_{1,2}$, and $\phi^{ \pm}$. But even in that case, we cannot do it for $\phi^{0}$ and Higgs boson.

\section{Conclusion}

The left right twin Higgs model is a concrete realization of the twin Higgs mechanism. The model predicts a heavy top quark, heavy gauge and various scalar bosons with a light SM Higgs boson, and thereby can yield rich phenomenology of the new particles at the LHC. The new heavy fermion and scalars also contribute significantly to the isospin violating $\rho$ parameter. We find that a one-loop analysis of $\rho$-parameter reduce parameter space of the model and set rough bounds for the masses of the heavy particles. In particular, we demonstrated that symmetry breaking parameter $f$ can be either smaller than $660 \mathrm{GeV}$ or lager than $1.1 \mathrm{TeV}$, and is the most crucial among the parameters to study the model. Analysis of other one-loop processes as well as study of collider physics is mandatory to reduce the region of parameter space further. Future work will include other oblique parameters.

\section{Acknowledgments}

We wish to thank Professor Pyungwon Ko for fruitful comments.

\section{A. Coupling constant in the LRTH model}

In the appendix we give a summary of the relevant coupling constants for our calculation. 
The gauge-fermion interaction is given by

$$
\begin{aligned}
\mathcal{L} & =i \bar{\psi}_{1} \gamma_{\mu}\left(g_{V}+g_{A} \gamma_{5}\right) \psi_{2} X^{\mu} \\
& =i \bar{\psi}_{1} \gamma_{\mu}\left(c_{L} P_{L}+c_{R} P_{R}\right) \psi_{2} X^{\mu}
\end{aligned}
$$

where $P_{L, R}=\frac{1}{2}\left(1 \mp \gamma_{5}\right)$ are the projection operators. We tabulate the gauge coupling constants of the fermions in Table 1. The gauge-scalar interaction is also relevant to our calculation. We choose the unitary gauge where all gauge-scalar mixing terms vanish. The various gauge coupling constants of the scalar fields are given in Table 2, 3, and 4 .

\section{B. One-loop integrals}

In this appendix we list scalar integrals involved with one-loop Feynman diagrams. The oneloop scalar integrals are decomposed in terms of Passarino-Veltman functions [22] which are defined in $n=4-2 \epsilon$ dimensions,

$$
\begin{aligned}
& Q^{4-n} \int \frac{d^{n} k}{(2 \pi)^{n}} \frac{1}{k^{2}-m^{2}+i \epsilon} \equiv \frac{i}{16 \pi^{2}} A_{0}\left(m^{2}\right), \\
& Q^{4-n} \int \frac{d^{n} k}{(2 \pi)^{n}} \frac{1}{\left(k^{2}-m_{1}^{2}+i \epsilon\right)\left((k-p)^{2}-m_{2}^{2}+i \epsilon\right)} \equiv \frac{i}{16 \pi^{2}} B_{0}\left(p^{2}, m_{1}^{2}, m_{2}^{2}\right), \\
& Q^{4-n} \int \frac{d^{n} k}{(2 \pi)^{n}} \frac{k_{\mu}}{\left(k^{2}-m_{1}^{2}+i \epsilon\right)\left((k-p)^{2}-m_{2}^{2}+i \epsilon\right)} \equiv \frac{i}{16 \pi^{2}} p_{\mu} B_{1}\left(p^{2}, m_{1}^{2}, m_{2}^{2}\right), \\
& Q^{4-n} \int \frac{d^{n} k}{(2 \pi)^{n}} \frac{k_{\mu} k_{\nu}}{\left(k^{2}-m_{1}^{2}+i \epsilon\right)\left((k-p)^{2}-m_{2}^{2}+i \epsilon\right)} \equiv \frac{i}{16 \pi^{2}}\left[g_{\mu \nu} B_{22}\left(p^{2}, m_{1}^{2}, m_{2}^{2}\right)\right. \\
&\left.+p_{\mu} p_{\nu} B_{11}\left(p^{2}, m_{1}^{2}, m_{2}^{2}\right)\right],
\end{aligned}
$$

where $1 / \hat{\epsilon}=(4 \pi)^{\epsilon} \Gamma(1+\epsilon) / \epsilon$. We also define the following integrals,

$$
\begin{aligned}
I_{1}(a) & =\int_{0}^{1} d x \ln [1-a x(1-x)], \\
I_{3}(a) & =\int_{0}^{1} d x x(1-x) \ln [1-a x(1-x)], \\
I_{4}(a, b) & =\int_{0}^{1} d x \ln [1-x+a x-b x(1-x)] .
\end{aligned}
$$

\section{Gauge boson self-energies in the LRTH model}

In this appendix, we compute the four gauge boson self-energies, $\Pi^{\gamma \gamma^{\prime}}(0), \Pi^{\gamma Z}\left(M_{Z}^{2}\right), \Pi^{W W}(0)$ and $\Pi^{Z Z}\left(M_{Z}^{2}\right)$. In general, the gauge independence in the bosonic sector can be retained by using the pinch technique or by using the background field method [23, 24]. In our calculations, there are three one-loop diagrams involved with an internal gauge boson propagator. In these diagrams we take only gauge invariant parts which is proportional to $\ln \left(M_{S}^{2}\right) / 16 \pi^{2}$. 


\section{C.1 Contributions to $\Pi^{\gamma \gamma^{\prime}}(0)$}

There are seven diagrams that contributes to $\Pi^{\gamma \gamma}$ in the LRTH model. These are loops having $(\bar{t} t),(\bar{b} b),(\bar{T} T),\left(\phi^{+} \phi^{-}\right)$and $\left(\hat{h}_{1}^{+} \hat{h}_{1}^{-}\right)$. The total contribution to $\Pi^{\gamma \gamma^{\prime}}(0)$ in the LRTH model is

$$
\Pi^{\gamma \gamma^{\prime}}(0)=\frac{\alpha}{4 \pi}\left[\frac{1}{3} \ln \frac{Q^{2}}{m_{\phi^{+}}^{2}}+\frac{1}{3} \ln \frac{Q^{2}}{m_{\hat{h}_{1}}^{2}}+\frac{16}{9} \ln \frac{Q^{2}}{m_{t}^{2}}+\frac{4}{9} \ln \frac{Q^{2}}{m_{b}^{2}}+\frac{16}{9} \ln \frac{Q^{2}}{m_{T}^{2}}+\frac{14}{3 \hat{\epsilon}}\right] .
$$

\section{C.2 Contributions to $\Pi^{\gamma Z}\left(M_{Z}^{2}\right)$}

There are seven diagrams that contributes to $\Pi^{\gamma Z}\left(M_{Z}^{2}\right)$ in the LRTH model. These are fermionic loops having $(\bar{t} t),(\bar{T} T)$ and $(\bar{b} b)$, the scalar loops due to $S S V$ coupling, $\left(\phi^{+} \phi^{-}\right)$, $\left(\hat{h}_{1}^{+} \hat{h}_{1}^{-}\right)$, and the $\phi^{+}$and $\hat{h}_{1}^{+}$scalar loops due to $S S V V$ quartic couplings. The contributions to $\Pi^{\gamma Z}\left(M_{Z}^{2}\right)$ due to the fermions are

$$
\begin{aligned}
& \Pi_{\bar{t} t}^{\gamma Z}\left(M_{Z}^{2}\right)=\frac{N_{c} \alpha}{\pi} \frac{2}{3 s_{w} c_{w}}\left(\frac{1}{2} C_{L}^{2}-\frac{4}{3} s_{w}^{2}\right) M_{Z}^{2}\left[\frac{1}{3}\left(\ln \frac{Q^{2}}{m_{t}^{2}}+\frac{1}{\hat{\epsilon}}\right)-2 I_{3}\left(\frac{M_{Z}^{2}}{m_{t}^{2}}\right)\right] \\
& \Pi_{\bar{T} T}^{\gamma Z}\left(M_{Z}^{2}\right)=\frac{N_{c} \alpha}{\pi} \frac{2}{3 s_{w} c_{w}}\left(\frac{1}{2} S_{L}^{2}-\frac{4}{3} s_{w}^{2}\right) M_{Z}^{2}\left[\frac{1}{3}\left(\ln \frac{Q^{2}}{m_{T}^{2}}+\frac{1}{\hat{\epsilon}}\right)-2 I_{3}\left(\frac{M_{Z}^{2}}{m_{T}^{2}}\right)\right] \\
& \Pi_{\bar{b} b}^{\gamma Z}\left(M_{Z}^{2}\right)=-\frac{N_{c} \alpha}{4 \pi} \frac{2}{3 s_{w} c_{w}}\left(\frac{1}{2}-\frac{2}{3} s_{w}^{2}\right) M_{Z}^{2}\left[\frac{1}{3}\left(\ln \frac{Q^{2}}{m_{b}^{2}}+\frac{1}{\hat{\epsilon}}\right)-2 I_{3}\left(\frac{M_{Z}^{2}}{m_{b}^{2}}\right)\right]
\end{aligned}
$$

The contributions of scalar loops to $\Pi^{\gamma Z}\left(M_{Z}^{2}\right)$ due to $S S V$ couplings are

$$
\begin{aligned}
\Pi_{\hat{h}_{1} \hat{h}_{1}}^{\gamma Z}\left(M_{Z}^{2}\right)= & -\frac{\alpha}{4 \pi} \frac{c 2_{w}^{2}}{c_{w} s_{w}}\left[\left(M_{\hat{h}_{1}}^{2}-\frac{1}{6} M_{Z}^{2}\right)\left(\ln \frac{Q^{2}}{M_{\hat{h}_{1}}^{2}}+\frac{1}{\hat{\epsilon}}\right)\right. \\
& \left.+\left(\frac{1}{6} M_{Z}^{2}-\frac{2}{3} M_{\hat{h}_{1}}^{2}\right) I_{1}\left(\frac{M_{Z}^{2}}{M_{\hat{h}_{1}}^{2}}\right)+M_{\hat{h}_{1}}^{2}-\frac{1}{9} M_{Z}^{2}\right], \\
\Pi_{\phi^{+} \phi^{-}}^{\gamma Z}\left(M_{Z}^{2}\right)= & \frac{\alpha}{2 \pi} \frac{s_{w}}{c_{w}}\left[\left(M_{\phi^{+}}^{2}-\frac{1}{6} M_{Z}^{2}\right)\left(\ln \frac{Q^{2}}{M_{\phi^{+}}^{2}}+\frac{1}{\hat{\epsilon}}\right)\right. \\
& \left.+\left(\frac{1}{6} M_{Z}^{2}-\frac{2}{3} M_{\phi^{+}}^{2}\right) I_{1}\left(\frac{M_{Z}^{2}}{M_{\phi^{+}}^{2}}\right)+M_{\phi^{+}}^{2}-\frac{1}{9} M_{Z}^{2}\right] .
\end{aligned}
$$

The contributions of scalar loops to $\Pi^{\gamma Z}\left(M_{Z}^{2}\right)$ due to $S S V V$ couplings are

$$
\begin{gathered}
\Pi_{\hat{h}_{1} \hat{h}_{1}}^{\gamma Z}\left(M_{Z}^{2}\right)=\frac{\alpha}{4 \pi} \frac{c 2_{w}^{2}}{c_{w} s_{w}}\left[1+\ln \frac{Q^{2}}{M_{\hat{h}_{1}}^{2}}+\frac{1}{\hat{\epsilon}}\right] M_{\hat{h}_{1}}^{2}, \\
\Pi_{\phi^{+} \phi^{-}}^{\gamma Z}\left(M_{Z}^{2}\right)=-\frac{\alpha}{2 \pi} \frac{s_{w}}{c_{w}}\left[1+\ln \frac{Q^{2}}{M_{\phi^{+}}^{2}}+\frac{1}{\hat{\epsilon}}\right] M_{\phi^{+}}^{2} .
\end{gathered}
$$

The terms proportional to $M_{\hat{h}_{1}}^{2}$ and $M_{\hat{h}_{1}}^{2} \ln \left(Q^{2} / M_{\hat{h}_{1}}^{2}\right)$ in Eq. C.5) and (C.7) cancel between themselves and so do the terms proportional to $M_{\phi^{+}}^{2}$ and $M_{\phi^{+}}^{2} \ln \left(Q^{2} / M_{\phi^{+}}^{2}\right)$ in Eq. (C.6) and (C.8). For $p^{2}=0$, it can be easily checked that the total fermionic and scalar contributions vanish individually. Thus

$$
\Pi^{\gamma Z}(0)=0
$$

as expected in the unitary gauge. 


\section{C.3 Contributions to $\Pi^{W W}(0)$}

The contributions of fermion loops to $\Pi^{W W}(0)$ are given as follows,

$$
\begin{aligned}
& \Pi_{\bar{t} b}^{W W}(0)=-\frac{N_{c} \alpha}{4 \pi} \frac{C_{L}^{2}}{2 s_{w}^{2}} f_{1}\left(m_{t}^{2}, m_{b}^{2}\right), \\
& \Pi_{\bar{T} b}^{W W}(0)=-\frac{N_{c} \alpha}{4 \pi} \frac{S_{L}^{2}}{2 s_{w}^{2}} f_{1}\left(m_{T}^{2}, m_{b}^{2}\right),
\end{aligned}
$$

where $1 / \hat{\epsilon}$ terms are omitted, and $f_{1}\left(m_{1}^{2}, m_{2}^{2}\right)$ is defined as

$$
f_{1}\left(m_{1}^{2}, m_{2}^{2}\right)=\frac{1}{2}\left(m_{1}^{2}+m_{2}^{2}\right)+\frac{m_{1}^{4}}{m_{1}^{2}-m_{2}^{2}} \ln \frac{Q^{2}}{m_{1}^{2}}-\frac{m_{2}^{4}}{m_{1}^{2}-m_{2}^{2}} \ln \frac{Q^{2}}{m_{2}^{2}} .
$$

The contributions of scalar loops to $\Pi^{W W}(0)$ due to $S S V V$ couplings are given as follows,

$$
\begin{aligned}
& \Pi_{h}^{W W}(0)=\frac{\alpha}{16 \pi} \frac{1}{s_{w}^{2}}\left[1+\ln \frac{Q^{2}}{M_{h}^{2}}+\frac{1}{\hat{\epsilon}}\right] M_{h}^{2}, \\
& \Pi_{\hat{h}_{1}^{+}}^{W W}(0)=\frac{\alpha}{8 \pi} \frac{1}{s_{w}^{2}}\left[1+\ln \frac{Q^{2}}{M_{\hat{h}_{1}}^{2}}+\frac{1}{\hat{\epsilon}}\right] M_{\hat{h}_{1}}^{2}, \\
& \Pi_{\hat{h}_{2}^{0}}^{W W}(0)=\frac{\alpha}{8 \pi} \frac{1}{s_{w}^{2}}\left[1+\ln \frac{Q^{2}}{M_{\hat{h}_{2}}^{2}}+\frac{1}{\hat{\epsilon}}\right] M_{\hat{h}_{2}}^{2}, \\
& \Pi_{\phi^{+}}^{W W}(0)=-\frac{\alpha}{24 \pi} \frac{x^{2}}{s_{w}^{2}}\left[1+\ln \frac{Q^{2}}{M_{\phi^{+}}^{2}}+\frac{1}{\hat{\epsilon}}\right] M_{\phi^{+}}^{2}, \\
& \Pi_{\phi^{0}}^{W W}(0)=-\frac{\alpha}{432 \pi} \frac{x^{2}}{s_{w}^{2}}\left[1+\ln \frac{Q^{2}}{M_{\phi^{0}}^{2}}+\frac{1}{\hat{\epsilon}}\right] M_{\phi^{0}}^{2} .
\end{aligned}
$$

The contribution of scalar loops to $\Pi^{W W}(0)$ due to $S S V$ couplings has the following form

$$
\Pi_{\hat{h}_{1} \hat{h}_{2}}^{W W}(0)=-\frac{\alpha}{2 \pi} \frac{1}{s_{w}^{2}} g_{1}\left(M_{\hat{h}_{1}}^{2}, M_{\hat{h}_{2}}^{2}\right),
$$

where $g_{1}\left(m_{1}^{2}, m_{2}^{2}\right)$ is defined as

$$
g_{1}\left(m_{1}^{2}, m_{2}^{2}\right)=\frac{3}{8}\left(m_{1}^{2}+m_{2}^{2}\right)+\frac{1}{4\left(m_{1}^{2}-m_{2}^{2}\right)}\left[m_{1}^{4} \ln \frac{Q^{2}}{m_{1}^{2}}-m_{2}^{4} \ln \frac{Q^{2}}{m_{2}^{2}}\right] .
$$

The terms proportional to $M_{\hat{h}_{1}}^{2}$ and $M_{\hat{h}_{1}}^{2} \ln \left(Q^{2} / M_{\hat{h}_{1}}^{2}\right)$ in Eq. (C.14) and (C.18) cancel partially between themselves and so do the terms proportional to $M_{\hat{h}_{2}}^{2}$ and $M_{\hat{h}_{2}}^{2} \ln \left(Q^{2} / M_{\hat{h}_{2}}^{2}\right)$ in Eq. C.15) and (C.18). Although the terms proportional to $M_{\phi^{+, 0}}^{2}$ and $M_{\phi^{+, 0}}^{2} \ln \left(Q^{2} / M_{\phi^{+, 0}}^{2}\right)$ in Eq. (C.16) and (C.17) do not cancel out, their coefficients are significantly small and so are their contributions to $\Pi^{W W}(0)$.

The contributions of scalar-gauge-bosons loops to $\Pi^{W W}(0)$ have the following form

$$
\begin{aligned}
\Pi_{h W}^{W W}(0)= & \frac{\alpha}{4 \pi} \frac{M_{W}^{2}}{s_{w}^{2}}\left[\frac{5}{8}-\frac{3}{8} \frac{M_{h}^{2}}{M_{W}^{2}}+\frac{3}{4} \frac{M_{h}^{2}}{M_{W}^{2}-M_{h}^{2}} \ln \frac{Q^{2}}{M_{W}^{2}}\right. \\
& \left.+\frac{M_{h}^{2}}{M_{W}^{2}-M_{h}^{2}}\left(-1+\frac{M_{h}^{2}}{M_{W}^{2}}\right) \ln \frac{Q^{2}}{M_{h}^{2}}+\left(1-\frac{M_{W}^{2}+M_{h}^{2}}{4 M_{W}^{2}}\right) \frac{1}{\hat{\epsilon}}\right]
\end{aligned}
$$


We take only the contribution proportional to $\ln \left(M_{S}^{2}\right) / 16 \pi^{2}$, which is gauge invariant,

$$
\Pi_{h W}^{W W}(0)=\frac{\alpha}{4 \pi} \frac{M_{W}^{2}}{s_{w}^{2}}\left[\frac{M_{h}^{2}}{M_{W}^{2}-M_{h}^{2}}\left(-1+\frac{M_{h}^{2}}{M_{W}^{2}}\right) \ln \frac{Q^{2}}{M_{h}^{2}}\right] .
$$

\section{C.4 Contributions to $\Pi^{Z Z}\left(M_{Z}^{2}\right)$}

The complete list of fermionic contributions to the self-energy function $\Pi^{Z Z}\left(p^{2}\right)$ are given below.

$$
\begin{aligned}
\Pi_{(\bar{T} t)}^{Z Z}\left(M_{Z}^{2}\right)= & -\frac{N_{c}}{16 \pi^{2}}\left(\frac{e}{2 c_{w} s_{w}}\right)^{2}\left(C_{L}^{2} S_{L}^{2}+\frac{f^{2}}{\hat{f}^{2}} \frac{s_{w}^{4}}{c_{w}^{4}} x^{4} C_{R}^{2} S_{R}^{2}\right)\left[-\frac{1}{3 M_{Z}^{2}}\left(m_{T}^{2}-m_{t}^{2}\right)^{2}+\frac{2}{9} M_{Z}^{2}\right. \\
& +\frac{1}{6 M_{Z}^{2}}\left[-m_{T}^{4}+m_{t}^{4}-2 M_{Z}^{4}+M_{Z}^{2}\left(5 m_{T}^{2}+m_{t}^{2}\right)\right] \ln \frac{Q^{2}}{m_{T}^{2}} \\
& +\frac{1}{6 M_{Z}^{2}}\left[-m_{t}^{4}+m_{T}^{4}-2 M_{Z}^{4}+M_{Z}^{2}\left(5 m_{t}^{2}+m_{T}^{2}\right)\right] \ln \frac{Q^{2}}{m_{t}^{2}} \\
& -\frac{1}{6}\left[m_{t}^{2}+m_{T}^{2}-2 M_{Z}^{2}+\frac{\left(m_{t}^{2}-m_{T}^{2}\right)^{2}}{M_{Z}^{2}}\right]\left(I_{4}\left(\frac{m_{T}^{2}}{m_{t}^{2}}, \frac{M_{Z}^{2}}{m_{t}^{2}}\right)+I_{4}\left(\frac{m_{t}^{2}}{m_{T}^{2}}, \frac{M_{Z}^{2}}{m_{T}^{2}}\right)\right) \\
& \left.+\frac{1}{\hat{\epsilon}}\left[m_{T}^{2}+m_{t}^{2}-\frac{2}{3} M_{Z}^{2}\right]\right] \\
+ & \frac{N_{c}}{4 \pi^{2}}\left(\frac{e}{2 c_{w} s_{w}}\right)^{2}\left(C_{L} S_{L} C_{R} S_{R} \frac{f^{2}}{\hat{f}^{2}} \frac{s_{w}^{2}}{c_{w}^{2}} x^{2}\right) m_{t} m_{T}\left[\frac{1}{\hat{\epsilon}}+\ln \frac{Q^{2}}{m_{t}^{2}}-I_{4}\left(\frac{m_{T}^{2}}{m_{t}^{2}}, \frac{M_{Z}^{2}}{m_{t}^{2}}\right)\right] \\
\Pi_{(\bar{t} T)}^{Z Z}\left(M_{Z}^{2}\right)= & \Pi_{(\bar{T} t)}^{Z Z}\left(M_{Z}^{2}\right)\left(m_{t} \leftrightarrow m_{T}\right) \\
\Pi_{(\bar{t} t)}^{Z Z}\left(M_{Z}^{2}\right)= & -\frac{N_{c}}{16 \pi^{2}}\left(\frac{e}{2 c_{w} s_{w}}\right)^{2}\left[2\left(\left(\frac{1}{2} C_{L}^{2}-\frac{4}{3} s_{w}^{2}\right)^{2}+\frac{C_{L}^{4}}{4}\right) \mathcal{H}_{1}\left(m_{t}^{2}\right)\right. \\
- & \frac{16}{3} s_{w}^{2}\left(C_{L}^{2}-\frac{4}{3} s_{w}^{2}\right) \mathcal{H}_{2}\left(m_{t}^{2}\right) \\
+ & \left.\frac{1}{\hat{\epsilon}}\left[2\left(\left(\frac{1}{2} C_{L}^{2}-\frac{4}{3} s_{w}^{2}\right)^{2}+\frac{C_{L}^{4}}{4}\right)\left(2 m_{t}^{2}-\frac{2}{3} M_{Z}^{2}\right)+\frac{16}{3} s_{w}^{2}\left(C_{L}^{2}-\frac{4}{3} s_{w}^{2}\right) m_{t}^{2}\right]\right] \\
\Pi_{(\bar{b} b)}^{Z Z}\left(M_{Z}^{2}\right)= & -\frac{N_{c}}{16 \pi^{2}}\left(\frac{e}{2 c_{w} s_{w}}\right)^{2}\left[2\left(\left(\frac{1}{2}-\frac{2}{3} s_{w}^{2}\right)^{2}+\frac{1}{4}\right) \mathcal{H}_{1}\left(m_{b}^{2}\right)\right. \\
- & \frac{8}{3} s_{w}^{2}\left(C_{L}^{2}-\frac{4}{3} s_{w}^{2}\right) \mathcal{H}_{2}\left(m_{b}^{2}\right) \\
+ & \left.\frac{1}{\hat{\epsilon}}\left[2\left(\left(\frac{1}{2}-\frac{2}{3} s_{w}^{2}\right)^{2}+\frac{1}{4}\right)\left(2 m_{b}^{2}-\frac{2}{3} M_{Z}^{2}\right)+\frac{8}{3} s_{w}^{2}\left(1-\frac{2}{3} s_{w}^{2}\right) m_{b}^{2}\right]\right] \\
\Pi_{(\bar{T} T)}^{Z Z}\left(M_{Z}^{2}\right)= & -\frac{N_{c}}{16 \pi^{2}}\left(\frac{e}{2 c_{w} s_{w}}\right)^{2}\left[2\left(\left(\frac{1}{2} S_{L}^{2}-\frac{4}{3} s_{w}^{2}\right)^{2}+\frac{S_{L}^{4}}{4}\right) \mathcal{H}_{1}\left(m_{T}^{2}\right)\right. \\
- & \frac{16}{3} s_{w}^{2}\left(S_{L}^{2}-\frac{4}{3} s_{w}^{2}\right) \mathcal{H}_{2}\left(m_{T}^{2}\right) \\
+ & \left.\left.\frac{1}{\hat{\epsilon}}\left[\left(\frac{1}{2} S_{L}^{2}-\frac{4}{3} s_{w}^{2}\right)^{2}+\frac{S_{L}^{4}}{4}\right)\left(2 m_{T}^{2}-\frac{2}{3} M_{Z}^{2}\right)+\frac{16}{3} s_{w}^{2}\left(S_{L}^{2}-\frac{4}{3} s_{w}^{2}\right) m_{T}^{2}\right]\right]
\end{aligned}
$$


where $\mathcal{H}_{1}\left(m^{2}\right)$ and $\mathcal{H}_{2}\left(m^{2}\right)$ are defined as

$$
\begin{aligned}
& \mathcal{H}_{1}\left(m^{2}\right)=\left(2 m^{2}-\frac{2}{3} M_{Z}^{2}\right) \ln \frac{Q^{2}}{m^{2}}+\frac{2}{9} M_{Z}^{2}+\frac{2}{3}\left(M_{Z}^{2}-m^{2}\right) I_{1}\left(\frac{M_{Z}^{2}}{m^{2}}\right), \\
& \mathcal{H}_{2}\left(m^{2}\right)=m^{2} I_{1}\left(\frac{M_{Z}^{2}}{m^{2}}\right)-m^{2} \ln \frac{Q^{2}}{m^{2}} .
\end{aligned}
$$

The contributions of scalar loops to $\Pi^{Z Z}\left(M_{Z}^{2}\right)$ due to $S S V V$ couplings have the following form,

$$
\begin{aligned}
\Pi_{(h)}^{Z Z}\left(M_{Z}^{2}\right) & =\frac{\alpha}{16 \pi} \frac{1}{c_{w}^{2} s_{w}^{2}}\left[1+\ln \frac{Q^{2}}{M_{h}^{2}}+\frac{1}{\hat{\epsilon}}\right] M_{h}^{2}, \\
\Pi_{\left(\hat{h}_{1}\right)}^{Z Z}\left(M_{Z}^{2}\right) & =\frac{\alpha}{8 \pi} \frac{c 2_{w}^{4}}{c_{w}^{2} s_{w}^{2}}\left[1+\ln \frac{Q^{2}}{M_{\hat{h}_{1}}^{2}}+\frac{1}{\hat{\epsilon}}\right] M_{\hat{h}_{1}}^{2}, \\
\Pi_{\left(\hat{h}_{2}\right)}^{Z Z}\left(M_{Z}^{2}\right) & =\frac{\alpha}{8 \pi} \frac{1}{c_{w}^{2} s_{w}^{2}}\left[1+\ln \frac{Q^{2}}{M_{\hat{h}_{2}}^{2}}+\frac{1}{\hat{\epsilon}}\right] M_{\hat{h}_{2}}^{2}, \\
\Pi_{\left(\phi^{+}\right)}^{Z Z}\left(M_{Z}^{2}\right) & =\frac{\alpha}{2 \pi} \frac{s_{w}^{2}}{c_{w}^{2}}\left[1+\ln \frac{Q^{2}}{M_{\phi^{+}}^{2}}+\frac{1}{\hat{\epsilon}}\right] M_{\phi^{+}}^{2}, \\
\Pi_{\left(\phi^{0}\right)}^{Z Z}\left(M_{Z}^{2}\right) & =-\frac{\alpha}{8 \pi} \frac{x^{2}}{54 c_{w}^{2} s_{w}^{2}}\left[1+\ln \frac{Q^{2}}{M_{\phi^{0}}^{2}}+\frac{1}{\hat{\epsilon}}\right] M_{\phi^{0}}^{2} .
\end{aligned}
$$

The contributions of scalar loops to $\Pi^{Z Z}\left(M_{Z}^{2}\right)$ due to $S S V$ couplings have the following form,

$$
\begin{aligned}
\Pi_{\left(\hat{h}_{1}^{\dagger} \hat{h}_{1}\right)}^{Z Z}\left(M_{Z}^{2}\right)= & -\frac{\alpha}{8 \pi} \frac{c 2_{w}^{4}}{c_{w}^{2} s_{w}^{2}}\left[\left(M_{\hat{h}_{1}}^{2}-\frac{1}{6} M_{Z}^{2}\right)\left(\ln \frac{Q^{2}}{M_{\hat{h}_{1}}^{2}}+\frac{1}{\hat{\epsilon}}\right)\right. \\
& \left.+\left(\frac{1}{6} M_{Z}^{2}-\frac{2}{3} M_{\hat{h}_{1}}^{2}\right) I_{1}\left(\frac{M_{Z}^{2}}{M_{\hat{h}_{1}}^{2}}\right)+M_{\hat{h}_{1}}^{2}-\frac{1}{9} M_{Z}^{2}\right], \\
\Pi_{\left(\hat{h}_{2}^{\dagger} \hat{h}_{2}\right)}^{Z Z}\left(M_{Z}^{2}\right)= & -\frac{\alpha}{8 \pi} \frac{1}{c_{w}^{2} s_{w}^{2}}\left[\left(M_{\hat{h}_{2}}^{2}-\frac{1}{6} M_{Z}^{2}\right)\left(\ln \frac{Q^{2}}{M_{\hat{h}_{2}}^{2}}+\frac{1}{\hat{\epsilon}}\right)\right. \\
& \left.+\left(\frac{1}{6} M_{Z}^{2}-\frac{2}{3} M_{\hat{h}_{2}}^{2}\right) I_{1}\left(\frac{M_{Z}^{2}}{M_{\hat{h}_{2}}^{2}}\right)+M_{\hat{h}_{2}}^{2}-\frac{1}{9} M_{Z}^{2}\right], \\
\Pi_{\left(\phi^{+} \phi^{-}\right)}^{Z Z}\left(M_{Z}^{2}\right)= & -\frac{\alpha}{2 \pi} \frac{s_{w}^{2}}{c_{w}^{2}}\left[\left(M_{\phi^{+}}^{2}-\frac{1}{6} M_{Z}^{2}\right)\left(\ln \frac{Q^{2}}{M_{\phi^{+}}^{2}}+\frac{1}{\hat{\epsilon}}\right)\right. \\
& \left.+\left(\frac{1}{6} M_{Z}^{2}-\frac{2}{3} M_{\phi^{+}}^{2}\right) I_{1}\left(\frac{M_{Z}^{2}}{M_{\phi^{+}}^{2}}\right)+M_{\phi^{+}}^{2}-\frac{1}{9} M_{Z}^{2}\right], \\
\Pi_{\left(h \phi^{0}\right)}^{Z Z}\left(M_{Z}^{2}\right)= & 0 .
\end{aligned}
$$

The terms proportional to $M_{\hat{h}_{1}}^{2}$ and $M_{\hat{h}_{1}}^{2} \ln \left(Q^{2} / M_{\hat{h}_{1}}^{2}\right)$ in Eq. (C.31) and (C.35) cancel between themselves and so do the terms proportional to $M_{\hat{h}_{2}}^{2}$ and $M_{\hat{h}_{2}}^{2} \ln \left(Q^{2} / M_{\hat{h}_{2}}^{2}\right)$ in Eq. (C.32) and (C.36). The terms proportional to $M_{\phi^{+}}^{2}$ and $M_{\phi^{+}}^{2} \ln \left(Q^{2} / M_{\phi^{+}}^{2}\right)$ in Eq. (C.33) and (C.37) also cancel between themselves. 
There are contributions of scalar-gauge boson loops to $\Pi^{Z Z}\left(M_{Z}^{2}\right)$. We take only the contribution proportional to $\ln \left(M_{S}^{2}\right) / 16 \pi^{2}$, which is gauge invariant,

$$
\begin{aligned}
\Pi_{(Z h)}^{Z Z}\left(M_{Z}^{2}\right) & =\frac{\alpha}{8 \pi} \frac{M_{W}^{2}}{c_{w}^{4} s_{w}^{2}}\left[1-\frac{1}{12 M_{Z}^{2}}\left(3 M_{h}^{2}+2 M_{Z}^{2}\right)\right] \ln \frac{Q^{2}}{M_{h}^{2}} \\
\Pi_{\left(Z_{H} h\right)}^{Z Z}\left(M_{Z}^{2}\right) & =\frac{\alpha}{8 \pi} \frac{e^{2} f^{2} x^{2}}{2 c_{w}^{4} c 2_{w}^{2}}\left[1-\frac{1}{12 M_{Z_{H}}^{2}}\left(3 M_{h}^{2}+3 M_{Z_{H}}^{2}-M_{Z}^{2}\right)\right] \ln \frac{Q^{2}}{M_{h}^{2}} .
\end{aligned}
$$

\section{References}

[1] B. C. Allanach, A. Djouadi, J. L. Kneur, W. Porod and P. Slavich, JHEP 0409 (2004) 044 [arXiv:hep-ph/0406166].

[2] G. Degrassi, S. Heinemeyer, W. Hollik, P. Slavich and G. Weiglein, Eur. Phys. J. C 28 (2003) 133 [arXiv:hep-ph/0212020].

[3] H. E. Haber, Nucl. Phys. Proc. Suppl. 62 (1998) 469 [arXiv:hep-ph/9709450].

[4] H. Georgi and A. Pais, Phys. Rev. D 10 (1974) 539.

[5] D. B. Kaplan and H. Georgi, Phys. Lett. B 136 (1984) 183.

[6] N. Arkani-Hamed, A. G. Cohen and H. Georgi, Phys. Lett. B 513 (2001) 232 [arXiv:hep-ph/0105239].

[7] N. Arkani-Hamed, A. G. Cohen, T. Gregoire and J. G. Wacker, JHEP 0208 (2002) 020 [arXiv:hep-ph/0202089].

[8] N. Arkani-Hamed, A. G. Cohen, E. Katz, A. E. Nelson, T. Gregoire and J. G. Wacker, JHEP 0208 (2002) 021 [arXiv:hep-ph/0206020].

[9] N. Arkani-Hamed, A. G. Cohen, E. Katz and A. E. Nelson, JHEP 0207 (2002) 034 [arXiv:hep-ph/0206021].

[10] M. Schmaltz, Nucl. Phys. Proc. Suppl. 117 (2003) 40 [arXiv:hep-ph/0210415].

[11] Z. Chacko, H. S. Goh and R. Harnik, Phys. Rev. Lett. 96 (2006) 231802 [arXiv:hep-ph/0506256].

[12] Z. Chacko, H. S. Goh and R. Harnik, JHEP 0601 (2006) 108 [arXiv:hep-ph/0512088].

[13] H. S. Goh and S. Su, arXiv:hep-ph/0611015.

[14] M. C. Chen and S. Dawson, Phys. Rev. D 70 (2004) 015003 [arXiv:hep-ph/0311032].

[15] A. Sirlin, Phys. Rev. D 22 (1980) 971.

[16] T. Blank and W. Hollik, Nucl. Phys. B 514 (1998) 113 [arXiv:hep-ph/9703392].

[17] M. Czakon, M. Zralek and J. Gluza, Nucl. Phys. B 573 (2000) 57 [arXiv:hep-ph/9906356].

[18] W. M. Yao et al. [Particle Data Group], J. Phys. G 33 (2006) 1.

[19] G. Rodrigo, A. Santamaria and M. S. Bilenky, Phys. Rev. Lett. 79 (1997) 193 [arXiv:hep-ph/9703358].

[20] [ALEPH Collaboration], Phys. Rept. 427 (2006) 257 [arXiv:hep-ex/0509008]. 
[21] T. Han, H. E. Logan, B. McElrath and L. T. Wang, Phys. Rev. D 67 (2003) 095004 [arXiv:hep-ph/0301040].

[22] G. Passarino and M. J. G. Veltman, Nucl. Phys. B 160 (1979) 151.

[23] G. Degrassi and A. Sirlin, Phys. Rev. D 46 (1992) 3104.

[24] A. Denner, G. Weiglein and S. Dittmaier, Phys. Lett. B 333 (1994) 420 [arXiv:hep-ph/9406204].

[25] G. Beall, M. Bander and A. Soni, Phys. Rev. Lett. 48 (1982) 848.

[26] R. N. Mohapatra, G. Senjanovic and M. D. Tran, Phys. Rev. D 66 (2002) 010001.

[27] A. A. Affolder et al. [CDF Collaboration], Phys. Rev. Lett. 87 (2001) 231803 [arXiv:hep-ex/0107008].

[28] S. Abachi et al. [D0 Collaboration], Phys. Rev. Lett. 76 (1996) 3271 [arXiv:hep-ex/9512007].

[29] R. Barate et al. [LEP Working Group for Higgs boson searches], Phys. Lett. B 565 (2003) 61 [arXiv:hep-ex/0306033]. 


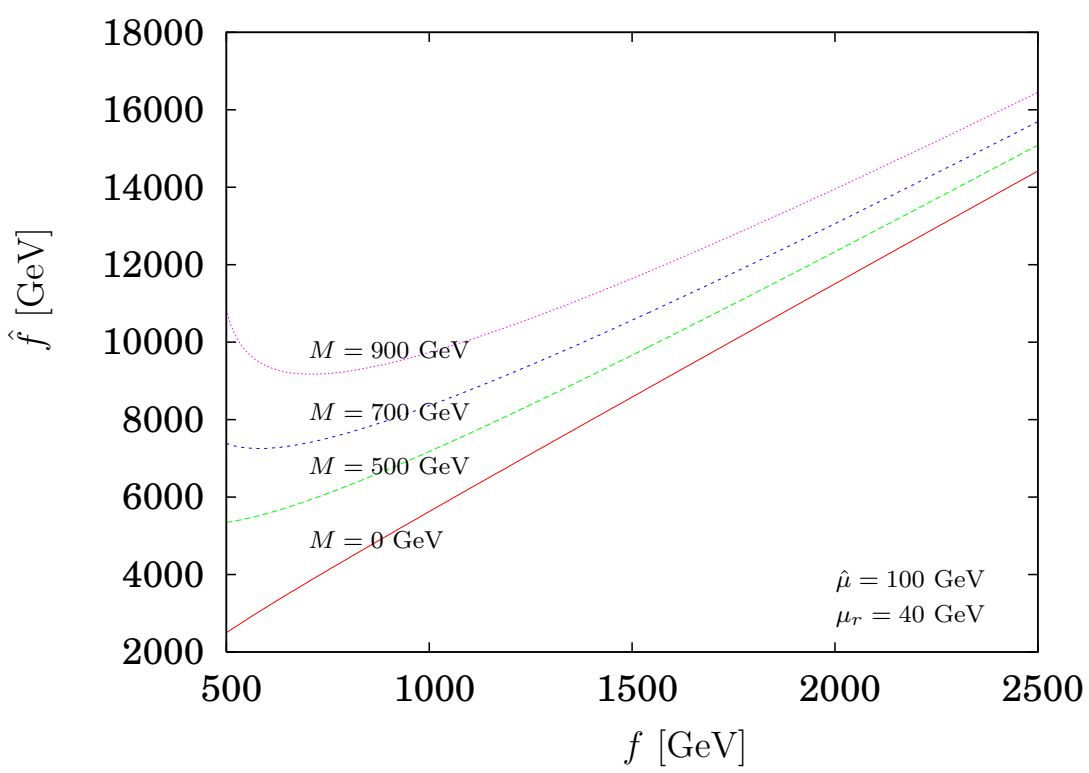

Figure 1: Plots of $\hat{f}$ versus $f$ with different values of $M$.

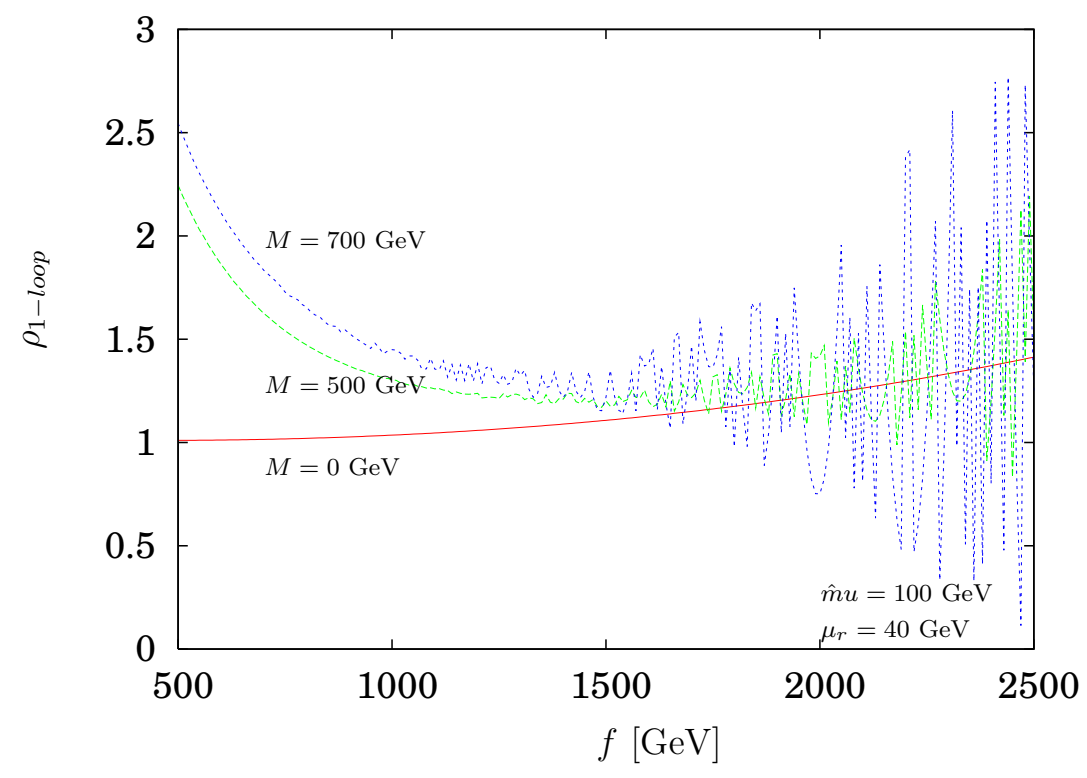

Figure 2: Plots of $\rho$-paramter versus $f$ with different values of $M$. 


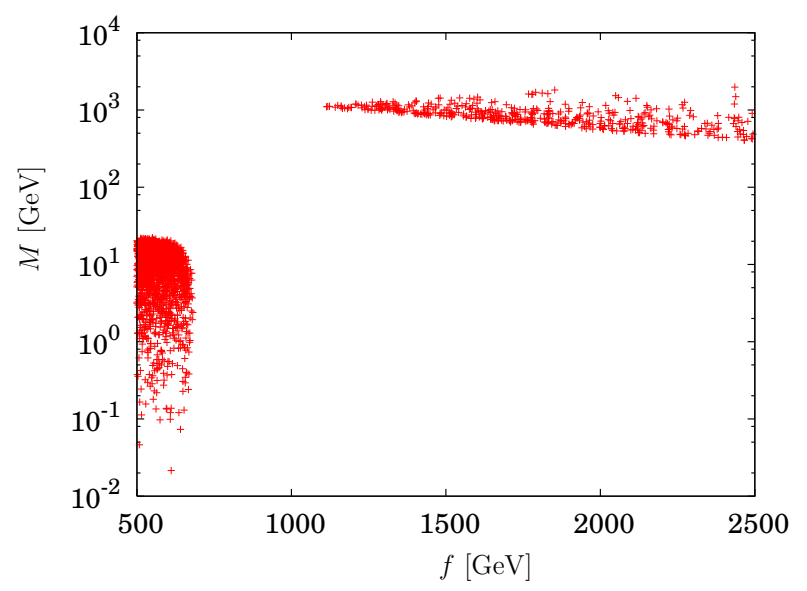

(a)

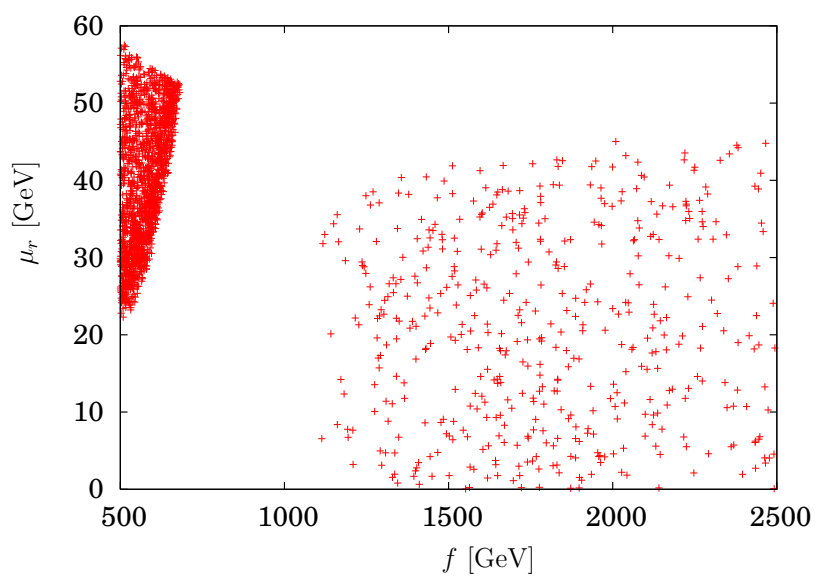

(b)

Figure 3: Allowed regions for (a) $M$ and (b) $\mu_{r}$.

\begin{tabular}{|c|c|c|}
\hline$X \bar{\psi} \psi$ & & \\
\hline$W \bar{t} b$ & $c_{L}=e C_{L} /\left(\sqrt{2} s_{w}\right)$ & $c_{R}=0$ \\
$W \bar{T} b$ & $c_{L}=e S_{L} /\left(\sqrt{2} s_{w}\right)$ & $c_{R}=0$ \\
\hline$Z \bar{t} t$ & $g_{V}=e\left(\frac{1}{4} C_{L}^{2}-\frac{2}{3} s_{w}^{2}\right) /\left(c_{w} s_{w}\right)$ & $g_{A}=-\frac{1}{4} e C_{L}^{2} /\left(c_{w} s_{w}\right)$ \\
$Z \bar{b} b$ & $g_{V}=e\left(-\frac{1}{2}+\frac{2}{3} s_{w}^{2}\right) /\left(2 c_{w} s_{w}\right)$ & $g_{A}=e /\left(4 c_{w} s_{w}\right)$ \\
$Z \bar{T} T$ & $g_{V}=e\left(\frac{1}{4} S_{L}^{2}-\frac{2}{3} s_{w}^{2}\right) /\left(c_{w} s_{w}\right)$ & $g_{A}=-\frac{1}{4} e S_{L}^{2} /\left(c_{w} s_{w}\right)$ \\
$Z \bar{T} t$ & $c_{L}=e C_{L} S_{L} /\left(2 c_{w} s_{w}\right)$ & $c_{R}=e f^{2} x^{2} s_{w} C_{R} S_{R} /\left(2 \hat{f}^{2} c_{w}^{3}\right)$ \\
\hline$A \bar{f} f$ & $g_{V}=e Q_{f}$ & $g_{A}=0$ \\
\hline
\end{tabular}

Table 1: Relevant coupling constants $X \bar{\psi} \psi$. The mixing angles $C_{L}=\cos \alpha_{L}, C_{R}=\cos \alpha_{R}$, etc are given in Eq. (2.20). 


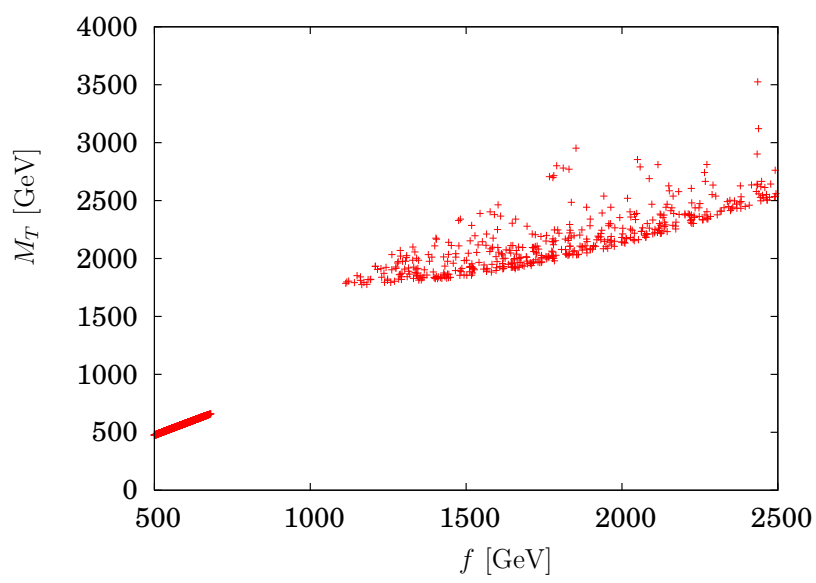

(a)

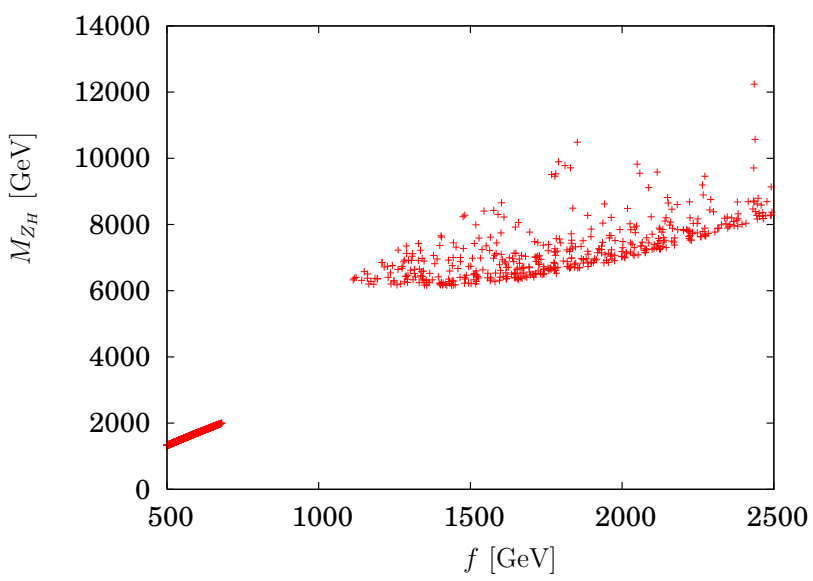

(b)

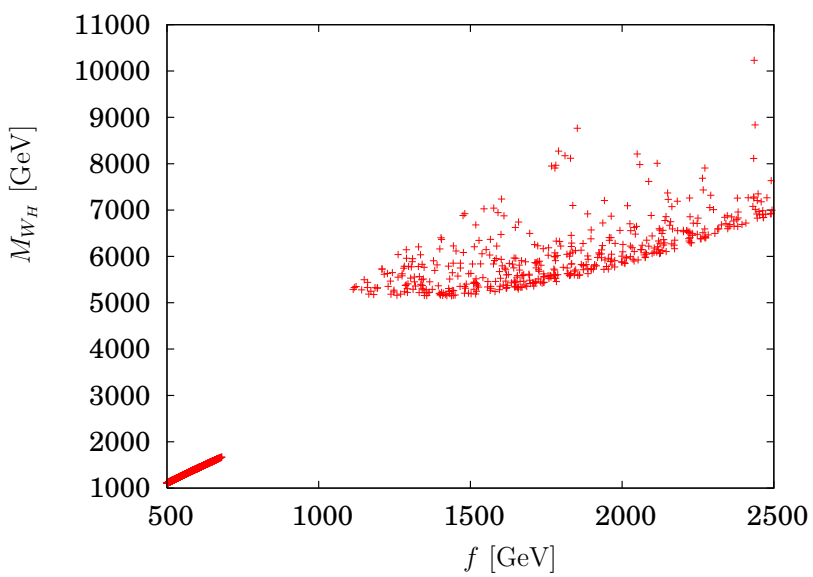

(c)

Figure 4: Masses for (a) heavy top, $M_{T}$, (b) heavy $Z, M_{Z_{H}}$, and (c) heavy $W, M_{W_{H}}$. 


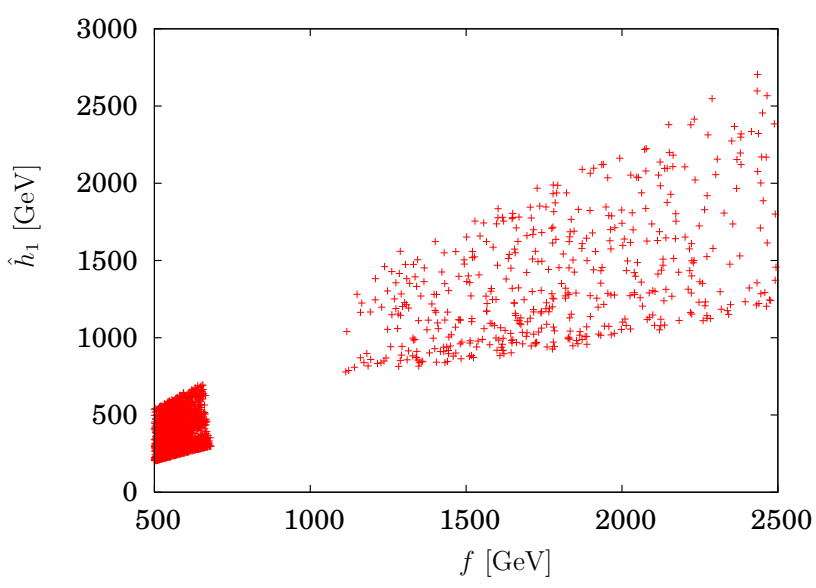

(a)

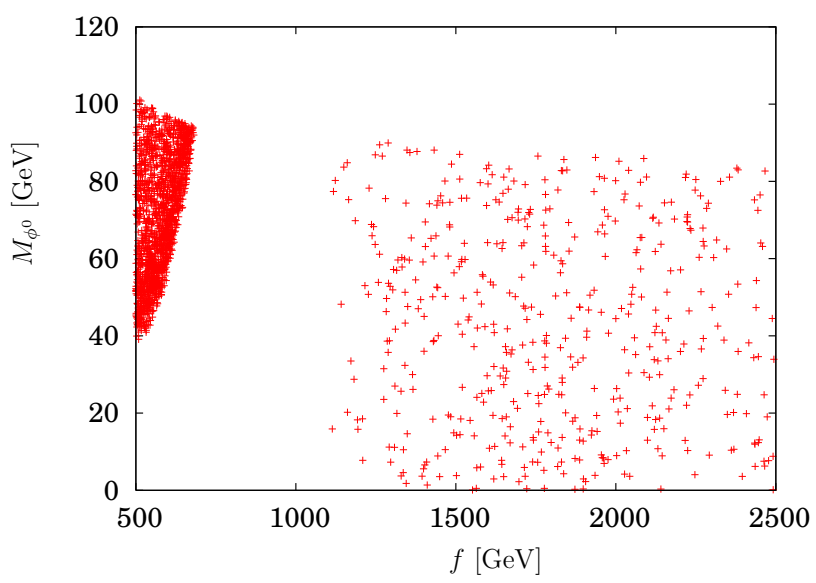

(b)

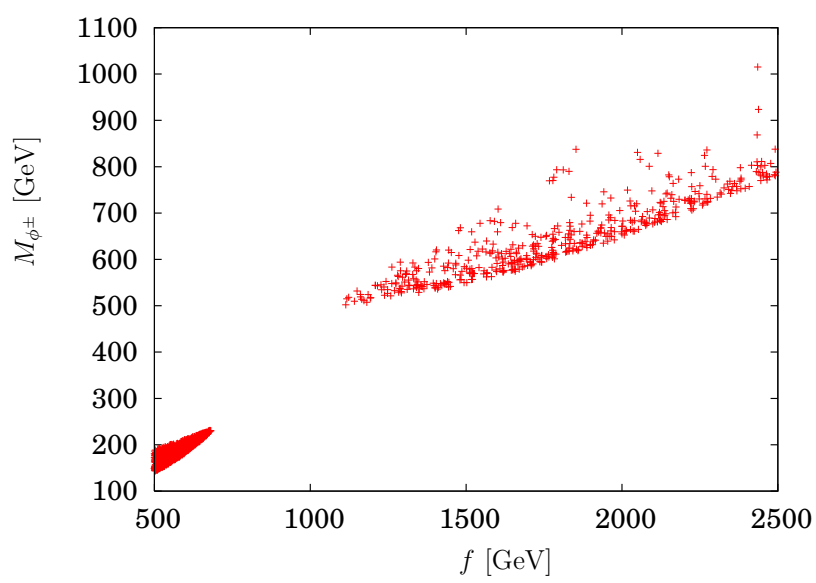

(c)

Figure 5: Masses for (a) $\hat{h}_{1}$, (b) $\phi^{0}$, and (c) $\phi^{ \pm} \cdot m_{\hat{h}_{2}}$ is almost same as $m_{\hat{h}_{1}}$ 


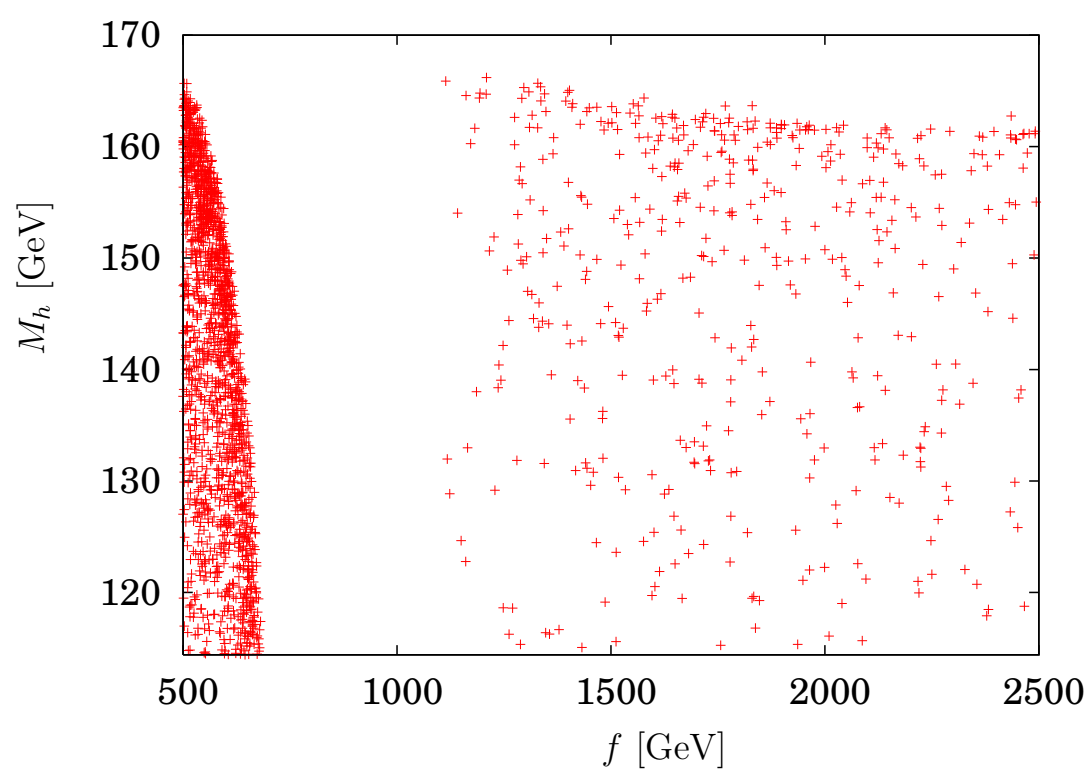

Figure 6: Higgs mass vs. $f$.

\begin{tabular}{|c|c|c|c|}
\hline$X X S S$ & $C_{X X S S}$ & $X X S S$ & $C_{X X S S}$ \\
\hline$W^{+} W^{-} h h$ & $e^{2} /\left(2 s_{w}^{2}\right)$ & $Z Z h h$ & $e^{2} /\left(2 c_{w}^{2} s_{2}^{2}\right)$ \\
$W^{+} W^{-} \phi^{0} \phi^{0}$ & $-e^{2} x^{2} /\left(54 s_{w}^{2}\right)$ & $Z Z \phi^{0} \phi^{0}$ & $-e^{2} x^{2} /\left(54 c_{w}^{2} s_{w}^{2}\right)$ \\
$W^{+} W^{-} \phi^{+} \phi^{-}$ & $-e^{2} x^{2} /\left(6 s_{w}^{2}\right)$ & $Z Z \phi^{+} \phi^{-}$ & $2 e^{2} s_{w}^{2} / c_{w}^{2}$ \\
$W^{+} W^{-} \hat{h}_{1}^{\dagger} \hat{h}_{1}$ & $e^{2} /\left(2 s_{w}^{2}\right)$ & $Z Z \hat{h}_{1}^{\dagger} \hat{h}_{1}$ & $e^{2} c 2_{w}^{4} /\left(2 c_{w}^{2} s_{w}^{2}\right)$ \\
$W^{+} W^{-} \hat{h}_{2}^{\dagger} \hat{h}_{2}$ & $e^{2} /\left(2 s_{w}^{2}\right)$ & $Z Z \hat{h}_{2}^{\dagger} \hat{h}_{2}$ & $e^{2} /\left(2 c_{w}^{2} s_{w}^{2}\right)$ \\
\hline$A A \hat{h}_{1}^{\dagger} \hat{h}_{1}$ & $2 e^{2}$ & $A A \phi^{+} \phi^{-}$ & $2 e^{2}$ \\
\hline$Z A \phi^{+} \phi^{-}$ & $-2 e^{2} s_{w} / c_{w}$ & $Z A \hat{h}_{1}^{\dagger} \hat{h}_{1}$ & $e^{2} c 2_{w}^{2} /\left(c_{w} s_{w}\right)$ \\
$Z W^{+} \hat{h}_{1}^{\dagger} \hat{h}_{2}$ & $-e^{2} /\left(\sqrt{2} c_{w}\right)$ & $Z W^{+} \hat{h}_{1}^{\dagger} \hat{h}_{2}$ & $e^{2} /\left(\sqrt{2} s_{w}\right)$ \\
\hline
\end{tabular}

Table 2: Relevant gauge coupling constants of the scalar fields, $C_{X X S S}$ 21].

\begin{tabular}{|c|c|c|c|}
\hline$X S S$ & $C_{X S S}$ & $X S S$ & $C_{X S S}$ \\
\hline$W^{+} \hat{h}_{1}^{\dagger} \hat{h}_{2}$ & $-e\left(p_{2}-p_{3}\right)_{\mu} /\left(\sqrt{2} s_{w}\right)$ & $A \hat{h}_{1}^{\dagger} \hat{h}_{1}$ & $-e\left(p_{2}-p_{3}\right)_{\mu}$ \\
$Z \hat{h}_{1}^{\dagger} \hat{h}_{1}$ & $-e\left(c_{w}^{2}-s_{w}^{2}\right)\left(p_{2}-p_{3}\right)_{\mu} /\left(2 c_{w} s_{w}\right)$ & $Z \hat{h}_{2}^{\dagger} \hat{h}_{2}$ & $e\left(p_{2}-p_{3}\right)_{\mu} /\left(2 c_{w} s_{w}\right)$ \\
$Z \phi^{-} \phi^{+}$ & $e\left(p_{2}-p_{3}\right)_{\mu} s_{w} / c_{w}$ & $A \phi^{-} \phi^{+}$ & $-e\left(p_{2}-p_{3}\right)_{\mu}$ \\
$Z h \phi^{0}$ & $i e x p_{1 \mu} /\left(6 c_{w} s_{w}\right)$ & & \\
\hline
\end{tabular}

Table 3: Relevant gauge coupling of the scalar fields, $C_{X S S} . p 1, p 2$ and $p 3$ refer to the incoming momentum of the first, second and third particle, respectively. [21. 


\begin{tabular}{|l|c|c|c|}
\hline$X_{1} X_{2} S$ & $C_{X_{1} X_{2} S}$ & $X_{1} X_{2} S$ & $C_{X_{1} X_{2} S}$ \\
\hline$W^{+} W^{-} h$ & $e M_{W} / s_{w}$ & $Z Z h$ & $e M_{W} /\left(c_{w}^{2} s_{w}\right)$ \\
$Z Z_{H} h$ & $e^{2} f x /\left(\sqrt{2} c_{w}^{2} c 2_{w}\right)$ & & \\
\hline
\end{tabular}

Table 4: Relevant gauge coupling constants of the scalar fields, $C_{X X S}$ [21].
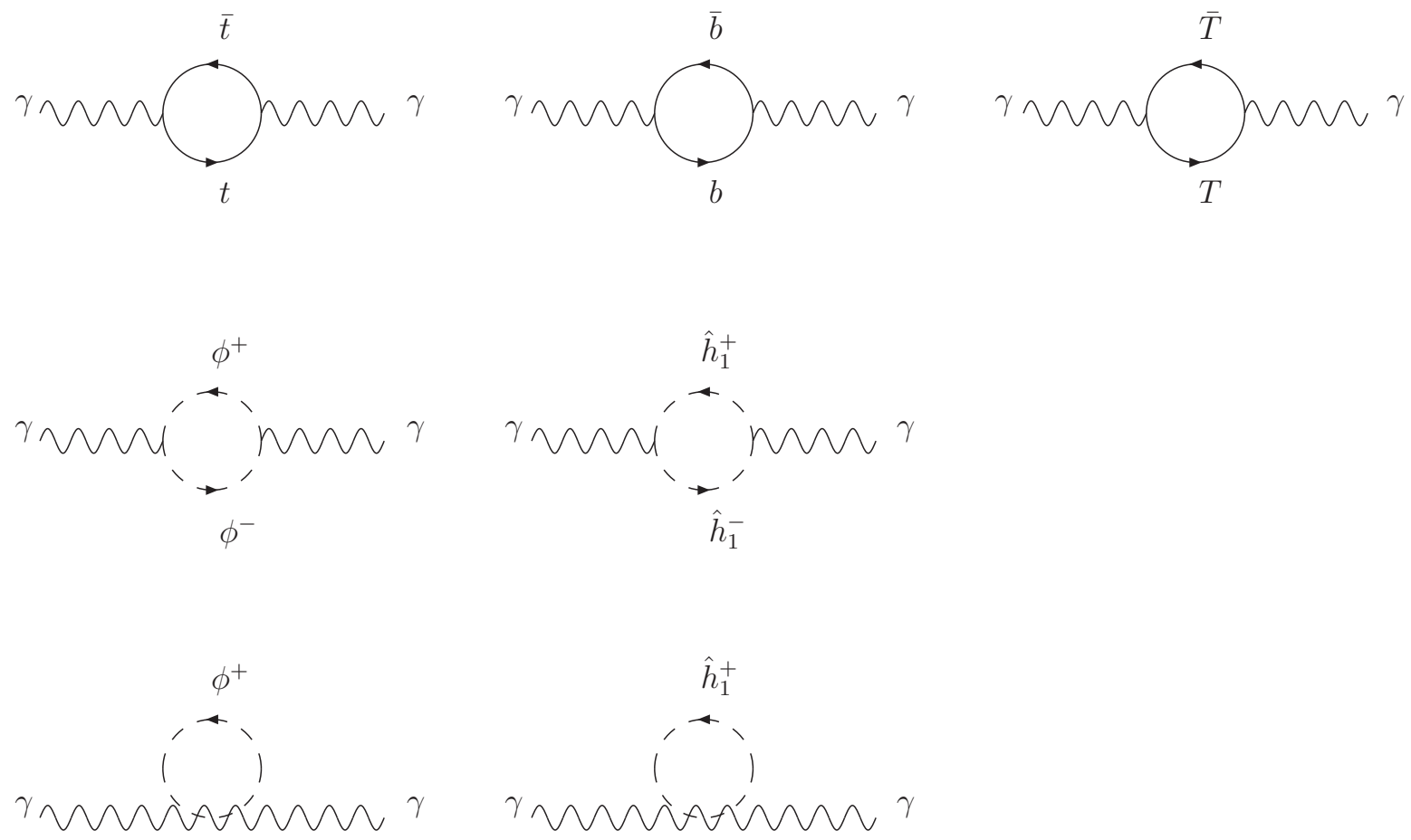

Figure 7: Complete list of diagrams due to fermion and scalar fields to the self-energy of the photon. 

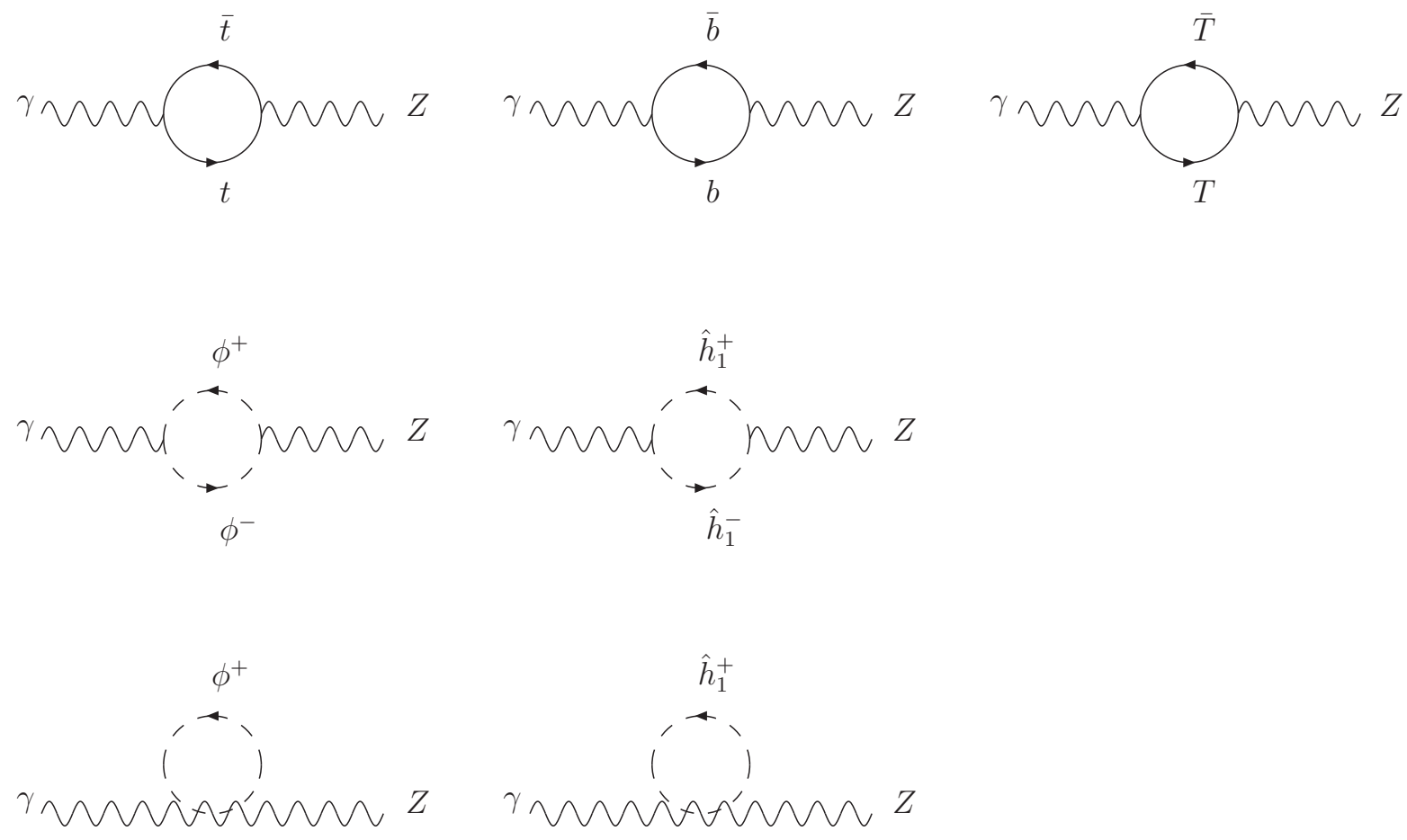

Figure 8: Complete list of diagrams due to fermion and scalar fields to the self-energy $\Pi^{\gamma Z}$. 

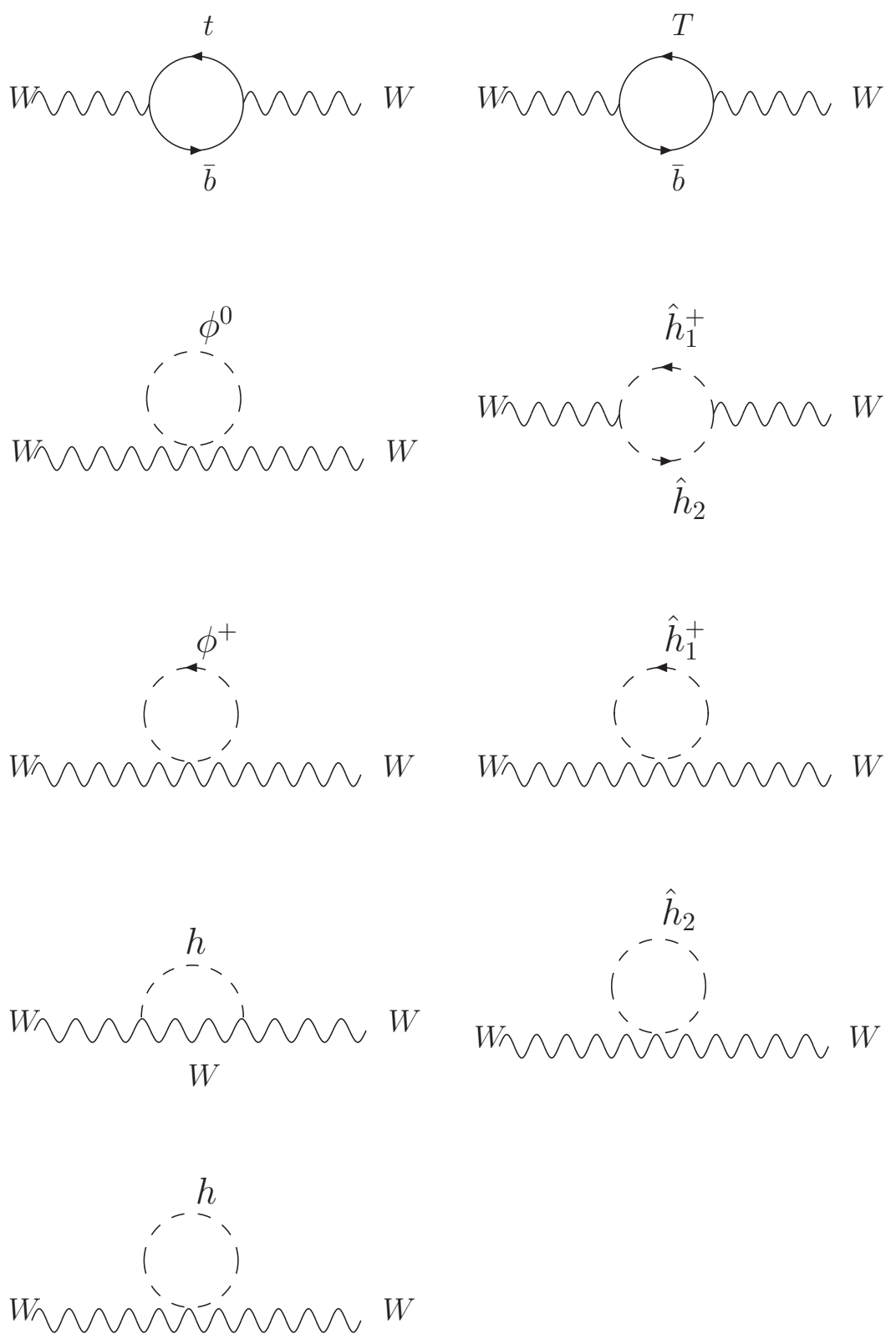

Figure 9: Complete list of diagrams due to fermion and scalar fields to the self-energy of the SM $W$ gauge boson. 

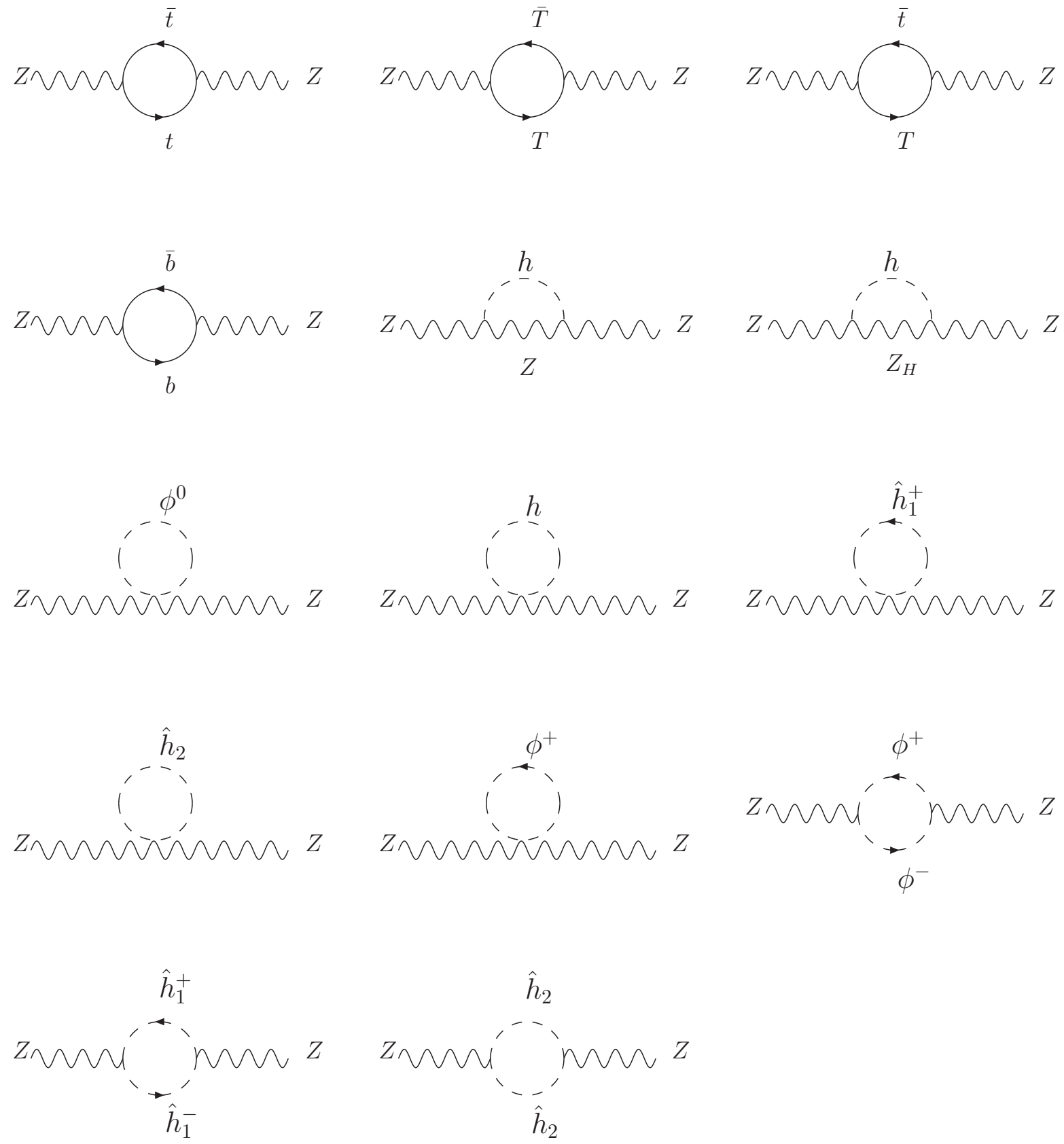

Figure 10: Complete list of diagrams due to fermion and scalar fields to the self-energy of the SM $Z$ gauge boson. 